\title{
Radiation driven bifurcations in fusion plasmas
}

\author{
S. Baschetti, D. Galassi, E. Serre \\ Aix Marseille Université, CNRS, Centrale Marseille, M2P2 UMR 7340, 13451, Marseille, France. \\ J. Bucalossi, H. Bufferand, G. Ciraolo, Ph. Ghendrih*, P. Tamain \\ CEA, IRFM, F-13108 Saint-Paul-lez-Durance, France \\ ${ }^{*}$ E-mail: philippe.ghendrih@cea.fr
}

September 9, 2016

\begin{abstract}
Operation of high performance fusion plasmas relies on self-organised properties to reach appropriate working points that are compatible with both high confinement performance to achieve a burning plasma, and controlled ageing of the confinement device. The latter conditions requires a trade-off between simplicity of the operation point and reaching conditions that can be sustained in steady state. The issue of heat flux control at the plasma edge and onto the plasma facing components is an example of this synergy. We address in this framework the problem of radiative divertor operation. The simplified 1D problem is recast in Hamiltonian formalism, the effective energy being invariant. This property is most efficient to address bifurcations and critical points leading to no-solution regions of the parameter space. Analytical investigation of these solutions indicates that taking into account the radiative front location and constraints on the upstream temperature reduces the operation space. Furthermore, one finds that radiative divertor operation tends to lead to operation at reduced plasma pressure, unless stable conditions and hot upstream plasma temperature can be sustained at vanishing divertor temperature.
\end{abstract}




\section{Contents}

1 Self-organisation in fusion plasmas: divertor control 3

2 The radiative divertor model $\quad 5$

2.1 Assumption: 1-D geometry . . . . . . . . . . . . . . . . 5

2.2 Divertor boundary conditions . . . . . . . . . . . . . . . 5

2.3 Assumption: vanishing ionisation layer . . . . . . . . . . . . 6

2.4 Assumption: constant total plasmas pressure . . . . . . . . . . . . 7

2.5 Control parameters: upstream conditions . . . . . . . . . . . . . 7

2.6 Energy balance and heat transport equations . . . . . . . . . . . . 8

2.7 Radiative divertor: normalised equations . . . . . . . . . . . . . . . . 9

3 Radiative divertor regimes $\quad 10$

3.1 Hot divertor, radiative divertor and cold SOL regimes . . . . . . . . . . . 10

3.2 Thermal bifurcations . . . . . . . . . . . . . . . . . . . 12

4 Properties of the Radiative divertor $\quad 16$

4.1 Location of the radiative front . . . . . . . . . . . . . . 16

4.2 Constraint on the upstream temperature . . . . . . . . . . . . . 17

4.3 Input power ramp-up at given radiative front position . . . . . . . . . . . . 19

4.4 Radiative efficiency . . . . . . . . . . . . . . . . . 20

4.5 Constrained operation due to the radiative bifurcations . . . . . . . . . 22

4.6 Density regimes with radiation . . . . . . . . . . . . . . 23

4.7 Constrained location of the radiative front in the divertor density regimes . 26

5 Controlled divertor operation $\quad 27$

5.1 Divertor regimes for different impurity species . . . . . . . . . . . . . 27

5.2 Width effect of the radiative cooling rate . . . . . . . . . . . . . 28

5.3 Optimised divertor operation . . . . . . . . . . . . . . . . . . 29

5.4 Dependence of divertor regimes on connection lengths . . . . . . . . . . 33

6 Discussion and conclusion $\quad 35$ 


\section{Self-organisation in fusion plasmas: divertor control}

Research on fusion plasmas will enter a new era when the tokamak ITER[9] starts operation. A tokamak is a particular geometry of the magnetic field that generates a configuration of plasma sustainment with axial symmetry and high confinement performance. When completed, ITER will allow one to rise the plasma thermal energy up to a range of $10 \mathrm{keV}$ to $20 \mathrm{keV}$, above the sun temperature, however at modest density, typically $10^{20} \mathrm{~m}^{-3}$. This will lead to a reasonable plasma pressure $\approx 3$ atmospheres $\left(3.210^{5} \mathrm{~Pa}\right)$. Magnetic confinement aims at ensuring the insulation of the hot plasma core and therefore maintain the plasma far from thermodynamical equilibrium with the surrounding components of the tokamak. Very large magnetic fields, typically $5 T$ in ITER, and large volumes of magnetised plasmas, $830 \mathrm{~m}^{3}$ for ITER, are required to reach sufficient insulation performance: namely to sustain a plasma at 100 million $K$ only $2 m$ away from solid state material at temperatures in the $1000 K$ range. If these target values are reached in ITER, the plasma will enter a burning regime such that direct heating power of the plasma by nuclear fusion reactions, about $100 M W$, will exceed the external heating power required to reach and sustain this state ${ }^{1}$, typically $50 \mathrm{MW}$. The plasma will then enter a phase of self-organised heating, a crucial demonstration on the way to considering fusion energy as a possible energy source.

In magnetic confinement devices, the motion of the charged particles that constitute the plasma, essentially ionised deuterium, a hydrogen isotope, and electrons experience a high frequency gyration motion with the magnetic field as axis. Confinement performance is thus governed by this constrained motion in the direction orthogonal to the magnetic field. Conversely, the motion along the magnetic field is close to that of free particles. This drives a very large anisotropy of transport between the direction parallel to the magnetic field and that transverse to the magnetic field. The confinement geometry of the magnetic field is completed by giving a helical structure to the field lines, winding it on nested toroidal surfaces. Parallel motion cannot lead to a displacement across these magnetic surfaces and therefore has no deleterious impact on confinement properties.

In practise, one finds that cross-field turbulence tends to reduce the insulation performance of this sophisticated magnetic field geometry. This deleterious effect governs the confinement performance and is therefore the main drive in determining the volume required for ITER : a torus with $6 \mathrm{~m}$ major radius and elliptical cross section of $4 \mathrm{~m} \times 8 \mathrm{~m}$, hence with a minimum distance of $2 \mathrm{~m}$ from the peak plasma temperature in the core to the boundary, or separatrix.

With such a size, ITER will demonstrate steady-state operation with respect to most of the characteristic evolution time constants of the plasma. The implication of this achievement is that the $150 \mathrm{MW}$ that will sustain the plasma at the required thermal energy will reach the wall of the plasma vessel and must then be transferred to cooling channels to maintain constant wall temperature. Such a transfer of energy, from plasma convection to heat diffusion in the wall material and then convection via the coolant out

\footnotetext{
${ }^{1}$ Note that $4 / 5$ of the nuclear power goes to the neutrons that are not coupled to the plasma, while $1 / 5$ goes to the helium nucleus. The latter channel is therefore the dominant heating source of ITER plasmas
} 
of the device, is presently one of the most severe operation issue for large tokamaks with burning plasmas. In such devices, the heating power scales like the plasma volume, while the deposition area scales at best like a surface. The problem being critical in ITER[7], it can only be more acute in the foreseen reactors. Indeed, the transport parallel to the magnetic field is so large compared to the transport across the magnetic field that when a wall component intersects a magnetic field line very large energy fluxes are then concentrated on this intersection point. The transition from the high confinement core plasma to the plasma in contact with the wall components is then found to take place on a very narrow boundary layer, in the millimetre range. With such geometrical features the energy flux flowing along the field line to the wall components will reach $3 \mathrm{GW} / \mathrm{m}^{2}$. This is equivalent to delivering the output power of three fission power plants on a single square metre. Alternatively, one can compare this number to the $70 \mathrm{MW} / \mathrm{m}^{2}$ energy out-flux at the sun surface.

Engineering skills allow one to extract up to $10 \mathrm{MW} / \mathrm{m}^{2}$, the gap between the $10 \mathrm{MW} / \mathrm{m}^{2}$ at the material surface and the $3 \mathrm{GW} / \mathrm{m}^{2}$ delivered by the plasma along field line must then be bridged by appropriate adjustment of the magnetic field and wall geometries as well as controlled transfer of energy from the confined plasma to particles that are not sensitive to the magnetic field, and thus escape the narrow channelling effect along the field lines onto the wall components. These particles are photons and neutral atoms, and the transfer process is therefore governed by atomic physics. Radiation, namely photon emission after excitation of impurity tied electron by electron impact, allows one to distribute the channelled electron energy flux to $4 \pi$ solid angle distribution, and therefore a much larger surface of plasma facing components, provided the emission takes place at sufficient geometrical distance from the component surfaces.

Atomic physics processes are quite sensitive to the plasma temperature and particularly to the electron temperature. For instance the cross section of deuterium atom ionisation exhibits a maximum at $20 \mathrm{eV}$ electron temperature. Depending on the temperature conditions, one must then consider different processes. At high temperature, as in the core plasma, only heavy impurities like tungsten, the chosen wall armour on ITER, can emit line radiation. In order to radiate in the outer and edge plasma, lighter impurities must be used, with a preference for noble gases to suppress deleterious chemical reactions. When the temperature is low enough, line radiation from hydrogen isotopes as well as equipartition between neutrals and ions can take place. Finally, the plasma can be extinguished towards the target plates in a recombining region dominated by neutral particles. This complex transition from the hot core plasma to the wall components with staged atomic processes that govern energy losses must be controlled. A dedicated volume within the confinement vessel is designed to optimise plasma-wall interaction: the divertor.

This paper addresses some aspects of the plasma self-organisation properties within the divertor volume due to radiation. The bifurcation aspect is driven by the condensation instability, first introduced in astrophysics. It can be summarised as an increase of the cooling efficiency as the temperature decreases. The simplified 1-D model is presented in Section 2. The different radiative divertor regimes are described in Section 3. and their main properties are analysed in Section 4. The role of radiation in controlling the divertor operation is discussed in Section 5. Discussion and conclusion Section 6, close 
the paper.

\section{The radiative divertor model}

In order to gain insight into the complex self-organisation of divertor plasmas, simplifications of the plasma model must be made to obtain analytical or near analytical models. These are presented here not only for completeness but also to discuss the assumptions used to to extract these simplified models.

\subsection{Assumption: 1-D geometry}

As outlined in Section 1, the heat flux in the parallel direction, flowing from the core plasma into the divertor region is several orders of magnitude too large compared to the conduction capability of solid state components. However, should the heat flux concentration governed by the very large parallel heat transport be alleviated, a surface of $15 \mathrm{~m}^{2}$ out of the $700 \mathrm{~m}^{2}$ surface of the plasma is sufficient to accommodate all the power in ITER at the limit of $10 \mathrm{MWm}^{-2}$ imposed by the present technology. Energy transfer to photons, which are not sensitive to magnetic field confinement, appears as a viable means to reduce the heat flux to the wall components provided the radiation process takes place at a sufficient distance from the vessel wall to allow photon energy deposition on a large enough surface. In order to address this problem we consider a 1-D model of heat transport along the magnetic field direction including radiative losses and appropriate boundary conditions.

\subsection{Divertor boundary conditions}

At the divertor wall, one must consider the so-called Bohm boundary condition which stipulates that the Mach number $M_{\text {div }}$ along the flow onto the wall must be supersonic[4], hence $M_{d i v}^{2} \geq 1$. The subscript div refers to the boundary value at the wall in the divertor volume. This location is equipped with specific components, the target plates, that are designed to sustain large heat fluxes close to the technological limit. The sign of the Mach number is defined such that the plasma goes into the wall. This condition corresponds to a flow into the void which is justified by the fact that the plasma promptly recombines at the wall contact so that it is in fact destroyed there, which implies this Bohm boundary condition. Since plasma disappears at the wall contact, it is to be noted that only outward convective transport can take place, and that conductive transport must tend to zero at the boundary. A second set of boundary conditions then governs the dependences of the energy outflux from the plasma, written here for both the main ion species and the electrons, therefore:

$$
\begin{aligned}
\Gamma_{d i v} & =M_{d i v} n_{d i v} C_{d i v} \\
Q_{d i v}^{e} & =\gamma_{e} \Gamma_{d i v} T_{d i v}^{e} \\
Q_{d i v}^{i} & =\gamma_{i} \Gamma_{d i v} T_{d i v}^{i} \\
Q_{d i v} & =Q_{d i v}^{e}+Q_{d i v}^{i}=\gamma \Gamma_{d i v} T_{d i v}^{e}
\end{aligned}
$$

Eq. (1a) constrains the particle flux $\Gamma_{d i v}$ in terms of the density $n_{d i v}$, sound velocity $C_{d i v}$ and Mach number $M_{d i v}$ at the divertor wall. The energy flux expressions, eq.(1b) and eq.(1c) for the electron and the ion channels respectively, take the same form as the 

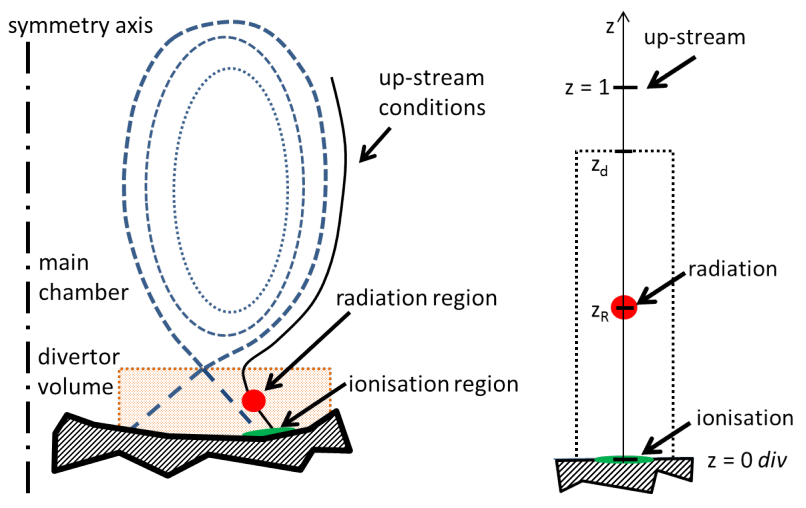

Figure 1: Sketch of the divertor region, left hand side geometry in a poloidal plane (cut of the axisymmetric torus at given toroidal angle), right hand side simplified geometry of the 1-D model.

total energy outflux eq.( $1 \mathrm{~d})$, namely a convective form proportional to the particle flux times the characteristic energy of the particles. The latter is assumed to be proportional to the thermal energies of the plasma species at the wall contact point, $T_{d i v}^{e}$ and $T_{d i v}^{i}$ for the electrons and ions respectively, with proportionality coefficient $\gamma_{e}, \gamma_{i}$. For the total energy flux, the coefficient is usually noted $\gamma$ and the reference energy is then the electron thermal energy[10] eq.(1d). These coefficients are determined theoretically from kinetic investigations of the distribution functions. Approximations of the latter can be computed analytically, however, empirical values appear as appropriate with $\gamma \approx 7$ and $\gamma_{e}>\gamma_{i}$ since the lost electrons are suprathermal in the parallel directions while the ion energy is typically the thermal energy in all directions of space. According to these expressions $\gamma=\gamma_{e}+T_{d i v}^{i} / T_{d i v}^{e} \gamma_{i}$. Note that these expressions do not assume the same thermal energy for the electrons and the ions and can be extended to cases where the parallel and transverse thermal energies are also different by considering that the thermal energies in eq.( 1 ) stand for parallel thermal energies and that the proportionality factors $\gamma_{e}$ and $\gamma_{i}$ depend on the ratios of the transverse to parallel thermal energies.

\subsection{Assumption: vanishing ionisation layer}

In the present 1-D plasma model we do not want to address the physics of the transition region between the conductive heat transport to the convective energy flux. We thus assume that the particle flux builds up in a very narrow region close to the wall surface by ionisation of neutral atoms leaving the wall surface. In all cases addressed in this paper, we neglect the energy loss due to the ionisation process and assume that an external parameter controlling the gas injection rate into the device allows one having an instantaneous feedback on the particle flux to the divertor target plate. Several examples will be discussed in the following. As a consequence of this very localised particle source, one can assume that the absolute value of the Mach number increases to unity within the ionisation layer and is small, hence such that $M^{2} \ll 1$, elsewhere along the 1-D geometry of the field line. 


\subsection{Assumption: constant total plasmas pressure}

Provided the momentum flux across fields lines and momentum exchange with neutral particles are negligible, one can readily show that the total plasma pressure is constant along field lines. Indeed, considering the total plasma momentum evolution equation, one finds that for a quasi-neutral plasma there is no effect of the mean electric field and that electron-ion momentum exchange does not contribute by definition. Mechanical balance for the plasma then yields that the sum of thermal and kinematic pressures for electron and ions, $\Pi$, is constant along the field lines.

$$
\Pi=n\left(T_{e}+T_{i}\right)+m_{i} n u_{i}^{2}=n\left(T_{e}+T_{i}\right)\left(1+M^{2}\right)
$$

This expression is obtained when neglecting the electron kinematic pressure $m_{e} n u_{e}^{2} \ll$ $m_{i} n u_{i}^{2}$ whenever $u_{e} \approx u_{i}$ in the vanishing mass ratio limit and assuming that the sound velocity $C_{s}$ is that of the constant temperature limit, the polytropic index is equal to one for both species, hence $m_{i} C_{s}^{2}=T_{e}+T_{i}$. This physics has moderate impact in the present problem since the thin ionisation layer leads to $M^{2} \ll 1$ in the whole domain, hence $\Pi=n\left(T_{e}+T_{i}\right)$, except for the boundary value $M_{d i v}^{2}$ that is larger or equal to 1 according to the Bohm boundary condition, hence $\Pi_{d i v}=n_{\text {div }}\left(T_{d i v}^{e}+T_{d i v}^{i}\right)\left(1+M_{d i v}^{2}\right)$. Given the constant pressure assumption, one then has $\Pi=\Pi_{d i v}$ so that for the standard Bohm boundary condition $M_{d i v}^{2}=1$

$$
\Pi=n\left(T_{e}+T_{i}\right)=2 n_{d i v}\left(T_{d i v}^{e}+T_{d i v}^{i}\right)
$$

\subsection{Control parameters: upstream conditions}

A sketch of the tokamak geometry is presented on Fig. 1. On the left hand size the toroidal symmetry of the torus is used to only draw the cross section at given toroidal location. This section of the tokamak is the so-called poloidal cross-section. The volume is divided in two parts: the upper part is called the main chamber, while the lower part is the divertor. In the main chamber, in the core plasma, the helical winding of the field lines draws toroidal magnetic surfaces which leads to closed lines in the cross-section (ellipses in dashed lines of the left hand side of the sketch). Towards the outer part of the magnetic geometry, there is a change in topology from these magnetic surfaces to a separatrix with an X-point. Within the separatrix magnetic surfaces are effective, outside the field line intersect wall components and exhibit large transport parallel to the field lines onto the wall. The latter field lines with intersection with the wall are called open field lines. In the main chamber the region with open field lines is called the Scrape-Off Layer (SOL).

One assumes here that scrape-off layer field lines are loaded by heat outflux from the core plasma in a region called upstream. This energy flux then flows along the field line (in both direction from the source) towards the divertor volume. In the 1-D model, Fig. 1 right hand side, the heat flux input is thus located at the upstream position at $z=1$ and is then transported to the wall component along the field line. The region starting at $z=z_{d}$ is the divertor chamber which is connected to the upstream source via parallel transport in the SOL from $z=1$ down to $z=z_{d}$. We assume here that external features determine the separation between the divertor chamber and the main chamber. When mechanical closure by solid components is implemented, one can readily define $z_{d}$, otherwise the $\mathrm{X}$-point location is the appropriate reference position to define 
$z_{d}$. In order to separate the core plasma working point from the divertor regime, radiation should be located with $z \leq z_{d}$, hence with no radiation in the SOL. We are thus interested in a divertor operating point such that radiation by the appropriate impurity will take place between the divertor throat at $z=z_{d}$ and the divertor target plate at $z=0$.

At the latter location, the boundary conditions in eq.( 1) relate the various parameters at the divertor wall. The physics of heat transport and losses on the way to the wall are thus governed by constraints at both the upstream and downstream conditions. This leads to a matching issue that is readily in determining both analytical and numerical solutions.

The upstream values of density, temperature, etc. are assumed to be coupled to the core physics. They act therefore as interface boundary conditions between SOL and core plasma. Depending on the field of interest, the role of the interface is different. Regarding the heat flux, one considers that the source is the core plasma, so that the upstream value is a boundary constraint for the 1-D model of the radiative divertor. Conversely, the upstream density, which results from the solution of the radiative divertor and the particle fuelling in the ionisation layer, must be considered as a boundary condition for the core density profile.

\subsection{Energy balance and heat transport equations}

Let us now address the radiative divertor model with the energy balance equation taking into account the radiative losses. These are assumed to be described by a density of radiation function $\mathcal{L}_{z}$, or radiative cooling rate[1], which only depends on the electron temperature. This expression includes all the ionisation and recombination processes for all tied electrons of the impurity atoms. Implicitly, it also assumes that these atomic processes are faster than other competing physics such as transport, in the regime called coronal equilibrium[3].

Let $Q$ be the energy flux in the low Mach number limit, hence governed by conduction, $T_{e}$ be the electron thermal energy, assumed to be the same for ions and electrons $T_{i}=T_{e}$, $n$ and $n_{z}$ the plasma and impurity densities, one then finds that the plasma steady state response is governed by two equations, heat transport eq.( $4 \mathrm{~b})$ and energy balance eq.( $4 \mathrm{~b})$.

$$
\begin{aligned}
\frac{d T_{e}}{d s} & =-\frac{Q}{\kappa_{\|}^{e}} \\
\frac{d Q}{d s} & =-n n_{z} \mathcal{L}_{z}\left(T_{e}\right)
\end{aligned}
$$

where $s$ is the curvilinear abscissa along the field line. In eq. (4b), the heat diffusivity $\kappa_{\|}$is that of the electrons that is much larger than that of the ions. Combined to the assumption of a single temperature for all species, hence an assumption of immediate equipartition, one thus finds that the electron heat transport is the effective heat conduction of the plasma under these conditions. Given the collisional heat diffusivity eq.( 5 ), one finds that it only depends on the electron thermal energy according to:

$$
\kappa_{\|}^{e}=\kappa_{R}^{e}\left(\frac{T_{e}}{T_{R}}\right)^{5 / 2}=3.16 \frac{n_{e} T_{e}}{m_{e} \nu_{e i}}=\frac{3.16 v_{R} T_{R}^{2}}{\frac{4}{3} \pi \sqrt{\frac{2}{\pi}}\left(e^{2} /\left(4 \pi \varepsilon_{0}\right)\right)^{2} \log \Lambda}\left(\frac{T_{e}}{T_{R}}\right)^{5 / 2}
$$


where the subscript $R$ refers to a normalisation thermal energy $T_{R}$, and $m_{e} v_{R}^{2}=T_{R}$, $m_{e} v_{e}^{2}=T_{e}$. The frequency $\nu_{e i}$ is the Coulomb collision frequency between electron and ions, which is defined by:

$$
\nu_{e i}=\frac{4}{3} \pi \sqrt{\frac{2}{\pi}}\left(\frac{e^{2} /\left(4 \pi \varepsilon_{0}\right)}{T_{e}}\right)^{2} \log \Lambda n_{e} v_{t h e}
$$

In this expression, $\log \Lambda$ is the Coulomb logarithm that takes into account the range of electrostatic binary collisions and $\Lambda$ is the plasma parameter.

\subsection{Radiative divertor: normalised equations}

We now introduce $z$ the normalised curvilinear abscissa along the field line $s=L_{\|} z$ where $L_{\|}$is the total length so that $z$ varies from 0 at the divertor target plate to 1 at the upstream position, see Fig. 1 right hand side. We normalise the thermal energy by $T_{R}$ and define $U=T_{e} / T_{R}=T_{i} / T_{R}$. Given eq.( $\left.4 \mathrm{~b}\right)$ and the thermal energy dependence of the heat diffusivity eq.(5), one defines $X=U^{7 / 2}$ and the normalised parallel heat flux $Y=-Q / Q_{T}$ such that:

$$
Q_{T}=\frac{2}{7} \kappa_{R}^{e} \frac{T_{R}}{L_{\|}}
$$

Eq. (4) is then written as:

$$
\begin{aligned}
& \frac{d X}{d z}=Y \\
& \frac{d Y}{d z}=\mathcal{C}_{z}\left(\frac{2 n T_{e}}{\Pi_{T}}\right)^{2} \ell_{z}(U)
\end{aligned}
$$

where $\ell_{z}(U)$ is the normalised radiative function and $Q_{R}$ the characteristic radiative energy flux and $\mathcal{C}_{z}$ a control parameter akin to the impurity concentration $c_{z}=n_{z} / n$.

$$
\begin{aligned}
\mathcal{C}_{z} & =\frac{Q_{R}}{Q_{T}} \\
\ell_{z}(U) & =\left(\frac{1}{U}\right)^{2} \frac{\mathcal{L}_{z}\left(T_{e}\right)}{\mathcal{L}_{R}} \\
Q_{R} & =c_{Z} \Pi_{T}^{2} \frac{L_{\|} \mathcal{L}_{R}}{4 T_{R}^{2}}
\end{aligned}
$$

In these expressions we have introduced a normalisation for the radiation density function, $\mathcal{L}_{R}$, and for the total plasma pressure, namely $\Pi_{T}$. These coefficients will be defined at a later stage depending on simplifications that can then be made. A factor 2 in the plasma pressure $2 n T_{e}$ is also used to take into account the thermal pressure of both plasma species given $T_{e}=T_{i}$. With this choice of normalisation the boundary relation eq.(1) for the total energy flux $Q_{d i v}$ is then:

$$
Y_{d i v}=\frac{\left|Q_{d i v}\right|}{Q_{T}}=\frac{\gamma \Pi_{d i v} C_{d i v}}{4 Q_{T}} \frac{2 T_{d i v}^{e}}{T_{d i v}^{e}+T_{d i v}^{i}} \frac{2\left|M_{d i v}\right|}{1+M_{d i v}^{2}}
$$


where $C_{d i v}$ is the plasma sound velocity at the divertor location, $m_{i} C_{d i v}^{2}=T_{d i v}^{e}+T_{d i v}^{i}$ and $\Pi_{d i v}=n_{d i v}\left(T_{d i v}^{e}+T_{d i v}^{i}\right)\left(1+M_{d i v}^{2}\right)$ is the total pressure at the same location. In eq. (10), one finds that $Y_{d i v}$ is the product of three terms, the two last ones being equal to 1 , respectively when $T_{d i v}^{e}=T_{d i v}^{i}$ and when $\left|M_{d i v}\right|=1$, the working assumptions of the present Section. The main dependence of $Y_{\text {div }}$ is therefore the first term, which can be simplified by defining the normalisation pressure such that:

$$
Q_{T}=\gamma \frac{\Pi_{T}}{4} C_{R}
$$

with $m_{i} C_{R}^{2}=2 T_{R}$. Defining the control parameter $\mathcal{C}_{\pi}$ as

$$
\mathcal{C}_{\pi}=\frac{\Pi_{\text {div }}}{\Pi_{T}}
$$

the boundary condition eq. (10) is then further simplified so that the set of equations to be solved is:

$$
\begin{aligned}
\frac{d X}{d z} & =Y \\
\frac{d Y}{d z} & =\mathcal{C}_{z} \mathcal{C}_{\pi}^{2} \ell_{z}(X) \\
Y_{d i v} & =\mathcal{C}_{\pi} X_{d i v}^{1 / 7}
\end{aligned}
$$

\section{Radiative divertor regimes}

\subsection{Hot divertor, radiative divertor and cold SOL regimes}

The structure of radiating functions for light impurities is characterised by a main peak corresponding to impact electron energies in the range of 5 to $30 \mathrm{eV}$. One finds indeed that at low temperature, the energy transfer by electron impact is too small to induce excitation of tied electrons. Conversely, at large temperature, the light impurities are fully ionised so that no line excitation is possible. This description also indicates that radiative efficiency combines the impurity radiation capability, determined by the radiating function, times the energy transport, or equivalently the dissipative force times the velocity of the energy transfer. One can therefore conveniently introduce the radiative work[2] as:

$$
\begin{aligned}
w(X) & =\int_{X}^{+\infty} \ell_{z}(X) d X \\
W(X) & =\mathcal{C}_{z} \mathcal{C}_{\pi}^{2} w(X)
\end{aligned}
$$

One then defines the normalising constant $\mathcal{L}_{R}$ of the radiation density function $\mathcal{L}_{z}$, eq. (9b), such that $w(0)=1$. With this definition, the profile equations eq.(13a) and eq. (13b) take the form of Hamiltonian trajectories with conjugate coordinates $(X, Y)$ and Hamiltonian $H$.

$$
\begin{aligned}
\frac{d X}{d z} & =Y=\frac{\partial H}{\partial Y} \\
\frac{d Y}{d z} & =-\frac{\partial W}{\partial X}=-\frac{\partial H}{\partial X} \\
H & =\frac{1}{2} Y^{2}+W(X)
\end{aligned}
$$


One then readily determines the free streaming trajectory with $W(X)=0$, hence $X=$ $Y\left(z-z_{d i v}\right)+X_{d i v}$, and $Y=Y_{d i v}$, as well as the energy exchange between kinetic $\frac{1}{2} Y^{2}$ and potential $W(X)$ energies. Using the invariant $H$ as well as the upstream and divertor conditions eq.( $13 \mathrm{c})$, one can capture many key properties of this system, indeed:

$$
\frac{1}{2} Y_{u p}^{2}+W\left(X_{u p}\right)=\frac{1}{2} Y_{d i v}^{2}+W\left(X_{d i v}\right)=\frac{1}{2} \mathcal{C}_{\pi}^{2} X_{d i v}^{2 / 7}+W\left(X_{d i v}\right)
$$

where $Y_{u p}$ is the upstream forcing condition and a given control parameter. As a consequence when $W\left(X_{u p}\right)$ is negligible, $W\left(X_{u p}\right) \approx 0$, one can determine $X_{d i v}$ and therefore $Y_{d i v}$. These two results determine the working conditions of the divertor and are sufficient to discuss many properties of radiative divertors. For a peaked radiating function, the radiative work, eq.( $14 \mathrm{~b})$ takes the form of a step function, Fig. 2. At the radiation peak $X=X_{R}$, the second derivative of $w(X)$ vanishes which yields an inflexion point. For $X \geq X_{R 2}$, there is no radiation so that $w(X)$ tends to zero, for a similar reason $X \leq X_{R 1}$ leads to $w(X) \rightarrow 1$. The shape of the transition region $X_{R 1} \leq X \leq X_{R 2}$, which depends on many atom physics properties of the impurity, has in practise little impact on the key physics of interest and can therefore be considerably simplified without changing the results.

Given eq.( 16), one can determine the divertor conditions without computing the profiles. Three different regimes can then de defined.

- Hot divertor regime: $X_{d i v} \geq X_{R 2}$. This corresponds to a hot divertor regime such that the plasma thermal energy in the whole region of interest is too large and the fully stripped impurities cannot emit line radiation. One then has $Y_{d i v}=Y_{u p}$ since there is no radiative loss, hence:

$$
U_{d i v}=X_{d i v}^{2 / 7}=\left(\frac{Y_{u p}}{\mathcal{C}_{\pi}}\right)^{2}
$$

- Radiative divertor regime: $X_{d i v} \leq X_{R 1}$ and $X_{u p} \geq X_{R 2}$. This is the regime of interest since radiation is located in the divertor volume and does not extend to the upstream plasma.

$$
X_{\text {div }}^{2 / 7}=\left(\frac{Y_{u p}}{\mathcal{C}_{\pi}}\right)^{2}-2 \mathcal{C}_{z}=X_{*}^{2 / 7}-2 \mathcal{C}_{z}
$$

where $X_{*}^{1 / 7}=Y_{u p} / \mathcal{C}_{\pi}$ is the value of $X$ at the divertor target plate in the case of the free streaming solution, $\mathcal{C}_{z}=0$.

- Cold SOL regime: $X_{u p} \leq X_{R 1}$. In this case the thermal energy of the plasma is too small over the whole profile to generate radiative losses. The plasma is then cold in the divertor up to the upstream location, the region of highest temperature in the SOL, hence the name of this regime. One then recovers:

$$
X_{d i v}=X_{*}
$$

together with $Y_{d i v}=Y_{u p}$ as in the case $X_{d i v} \geq X_{R 2}$. 


\subsection{Thermal bifurcations}

The divertor temperature solutions determined for the divertor regime in eq.(17) are defined in terms of the control parameters $Y_{u p}$ and $\mathcal{C}_{\pi}$. To relate the three divertor regimes of eq. (17), one must also determine the upstream thermal energy $X_{u p}$. This is readily done in the cold SOL and hot divertor regimes.

$$
\begin{aligned}
X_{*} \geq X_{R 2} \quad ; \quad X_{d i v}=X_{*} \quad ; \quad X_{u p}=X_{*}+Y_{u p} \\
X_{u p} \leq X_{R 1} \quad ; \quad X_{d i v}=X_{*} \quad ; \quad X_{u p}=X_{*}+Y_{u p}
\end{aligned}
$$

Let us define $X_{R 1}$, the lower-temperature bound of the radiative density function such that $W_{R}\left(X_{R 1}\right)=0.99$ and $X_{R 2}$, the upper-temperature bound, such that $W_{R}\left(X_{R 2}\right)=0.01$. Using these values one can then determine the various radiative regimes of the divertor, see Fig. 3. The threshold in energy flux $Y_{u p}$ for the hot divertor $Y_{u p}^{\text {hot }}$, and cold SOL $Y_{u p}^{\text {cold }}$, regimes are defined by $\left(Y_{\text {up }}^{\text {hot }} / \mathcal{C}_{\pi}\right)^{7}=X_{R 2}$ and $\left(Y_{\text {up }}^{\text {cold }} / \mathcal{C}_{\pi}\right)^{7}+Y_{\text {up }}^{\text {cold }}=X_{R 1}$ respectively. One thus finds that for $Y_{u p} \geq Y_{u p}^{\text {hot }}, X_{d i v} \geq X_{R 2}$, which corresponds to the hot divertor regime, the transition point being determined by $X_{d i v}=X_{*}=X_{R 2}$, see Fig. 3. Conversely, for $Y_{u p} \leq Y_{u p}^{\text {cold }}, X_{u p} \leq X_{R 1}$. This corresponds to the cold SOL regime, the transition being defined by $X_{u p}=X_{*}+Y_{u p}=X_{R 1}$, Fig. 3. It is to be noted that, given $z_{d}$ the location of the transition from the divertor volume to the main chamber Fig. 1, one can also track the transition from the cold divertor regime into the cold SOL regime determined by $X_{d}=X_{*}+Y_{u p} z_{d}=X_{R 1}$ hence at a critical input heat flux of $Y_{u p}^{\text {cold }} / z_{d}, Y_{u p}^{\text {cold }}$ being the cold SOL threshold. Note that with $z_{d} \approx 0.3$ there is a factor 3 between these two critical values and therefore significant operation margin in terms of input heat flux.

One can thus identify a regime such that radiation is located in the divertor and SOL volumes. This regime is bounded by the cold SOL regime where radiation has moved into the core plasma, and the hot divertor regime, such that no line radiation is taken into account since all radiative losses must be localised in the thin ionisation boundary layer, located in the vicinity of the target plate. Both latter regimes are unfavourable for tokamak operation. The key control parameter of the system is readily defined after eq. (16) that yields the conserved Hamiltonian of the trajectories. The momentum variation $\Delta Y=Y_{u p}-Y_{d i v}$ is the drop in heat flux along the field line and is therefore a criterion to evaluate the divertor efficiency, in particular by decoupling upstream and target plate conditions. The temperature drop $X_{u p}-X_{d i v}$, or equivalently $U_{u p}-U_{d i v}$ is also of interest to evaluate the distance between the upstream and divertor conditions. Finally, one can introduce criteria in terms of the energy balance such as the variation in potential energy $\Delta w=w\left(X_{d i v}\right)-w\left(X_{u p}\right)$ as well as the variation in kinetic energy $\Delta\left(Y^{2} / \mathcal{C}_{\pi}^{2}\right)=U_{*}-U_{\text {div }}$. Relative criteria, ranging from 0 to 1 , are indicators used in the following.

$$
\begin{aligned}
\varepsilon_{Y} & =\frac{\Delta Y}{Y_{u p}}=\frac{Y_{u p}-Y_{d i v}}{Y_{u p}}=1-\left(1-\frac{2 \mathcal{C}_{z} \mathcal{C}_{\pi}^{2}}{Y_{u p}^{2}} \Delta_{w}\right)^{1 / 2} \\
\varepsilon_{U} & =\frac{\Delta_{U}}{U_{*}}==\frac{U_{*}-U_{d i v}}{U_{*}}
\end{aligned}
$$

The divertor regime of interest is such that $w\left(X_{u p}\right) \rightarrow 0$ so that radiation is confined to the divertor volume. For optimum operation, we also consider $w\left(X_{\text {div }}\right) \rightarrow 1$ so that $\Delta_{w} \rightarrow 1$. One then finds that the normalised drop in heat flux, $\varepsilon_{Y}$ does not depend 
on the thermal energy profile $U(z)=X(z)^{2 / 7}$ but only on the combination of control parameters $Y_{u p}$, the input heat flux, $\mathcal{C}_{z}$, akin to the impurity concentration, and $\mathcal{C}_{\pi}$ the plasma pressure. Criteria of divertor performance are therefore $\varepsilon_{Y} \approx 1$, to lower the heat flux onto the target plate, together with $\varepsilon_{U} \rightarrow 1$, to lower the plasma temperature at the target plate and thus reduce erosion of target material with respect to the no radiation case. Consistently, when localising radiation within the divertor volume, one requires $U_{u p}-U_{d i v} \geq \Delta U_{R}$, where $\Delta U_{R}$ is the characteristic width of the radiation density function, $\Delta U_{R}=U_{R 2}-U_{R 1}$.

One finds that $\varepsilon_{Y}=1$, the maximum value, is achieved for $Y_{\text {div }}=0$, hence for $2 \mathcal{C}_{z} \mathcal{C}_{\pi}^{2} / Y_{\text {up }}^{2}=$ 1 eq.(19a). When such a value is reached, the temperature gradient drops to zero so that the temperature variation along the profile is vanishingly small. This limit corresponds to the cold SOL regime, and bounds consequently $\varepsilon_{Y}$ in the radiative regime to values smaller than 1.

If one now assumes that the upstream thermal energy $X_{u p}$ is always larger than the upper radiation limit $X_{R 2}$, then:

$$
\begin{aligned}
U_{*} & =U_{d i v}+2 \mathcal{C}_{z} w\left(U_{\text {div }}\right) \\
Y_{u p}^{2} & =Y_{d i v}^{2}+2 \mathcal{C}_{\pi}^{2} \mathcal{C}_{z} w\left(U_{d i v}\right)
\end{aligned}
$$

This assumption discards the cold SOL regime, and only two divertor regimes are addressed: the hot divertor, $w\left(U_{\text {div }}\right) \rightarrow 0$, such that for each value of the control parameter $U_{*}$ there is only one solution for the divertor thermal energy, $U_{d i v}=U_{*}$, and the radiative divertor regime such that $w\left(U_{\text {div }}\right)$ increases to 1 . In this regime, multiple solutions are found for $U_{* 1} \leq U_{*} \leq U_{* 2}$, see Fig. 4. The critical values of $U_{*}$ depend on $\mathcal{C}_{z}$ that determines the height of the step function that is added to $U_{d i v}$ in eq.( 20). When reducing $U_{*}$ the system undergoes a transition from the hot divertor regime with one solution $U_{\text {div }} \approx U_{*}$, hence with no radiation, to a situation with 3 solutions: for a given value of $U_{*}$, in the range $U_{* 1} \leq U_{*} \leq U_{* 2}, 3$ solutions for $U_{d i v}$ are readily determined graphically, see Fig. 4. The one with largest divertor temperature belongs to the hot divertor regime. The following one when decreasing the divertor temperature is such that $d U_{*} / d U_{d i v}<0$ and is found to be unstable[2]. The third solution, belongs to the cold divertor regime. Finally, a limit is reached at the third critical value $U_{* 3}$ when $U_{d i v}=0$ is reached and no solutions can be sustained at too low value of $U_{*}$, hence too low input energy flux. The transition to the cold-SOL regime can occur prior to this critical value but cannot be addressed with the reduced system eq.( 20). Indeed, at some point in the neighbourhood of $U_{\text {div }}=0$, a transition such that $w\left(U_{u p}\right) \rightarrow 1$ and $\Delta_{w} \rightarrow 0$ can occur which drives the system back to the no-radiation limit $U_{d i v} \approx U_{*}$. As shown in previous papers[2], these radiative bifurcations require rather peaked radiative loss functions, which restricts their signature to rapid variations of divertor parameters[5]. Although these bifurcations do not appear to depend on turbulent transport properties, they exemplify some of the key features of the L-H transition[11], including more recent results related to tungsten wall operation with extrinsic light impurity[8]. 


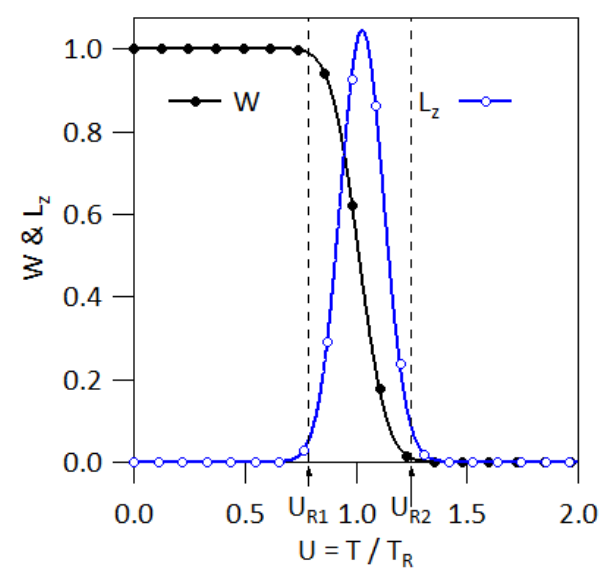

Figure 2: Sketch of the radiative cooling rate $L_{z}(X)$, open circles, and radiative work function $W(X)$, closed circles. The dashed vertical lines locate the lower, $U_{R 1}$, and upper, $U_{R 2}$, bound in terms of plasma thermal energy of line radiation for the particular impurity.

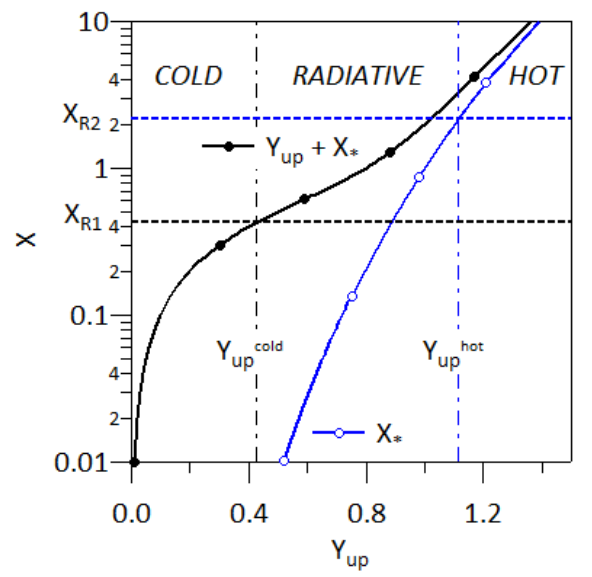

Figure 3: Divertor regimes identified by the transitions to the hot divertor regime, defined by the intersection of $X_{d i v}=X_{*}=X_{R 2}$ curve with open circle markers, and the transition to the cold SOL regime $X_{u p}=X_{*}+Y_{u p}=X_{R 1}$, curve with the closed circle markers. The intermediate regime is identified as the radiative divertor regime. 


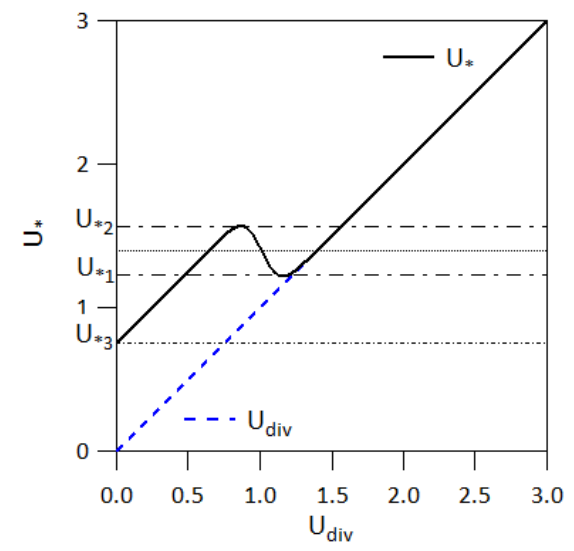

Figure 4: Divertor regimes $U_{\text {div }}\left(U_{*}\right)$ determined by $U_{*}\left(U_{\text {div }}\right)$, eq.( 20). The dashed line, $U_{d i v}=U_{*}$ corresponds to the no-radiation regime. Starting from high energy influx, $U_{*}$ large, in the hot divertor regime, and lowering $U_{*}$, the system exhibits a first bifurcation to a regime with 3 solutions at $U_{* 2}$, dash-dot horizontal line. At $U_{* 1}$ the system exhibits a bifurcation back to 1 solution, dash-dot horizontal line. At a third critical value, $U_{* 3}$, there is a limit transition to the case with no solutions, dash-dot horizontal line. The latter is specific of eq.(20), and, depending on conditions, can be replaced by a back transition to no-radiation in the cold SOL regime. 


\section{Properties of the Radiative divertor}

\subsection{Location of the radiative front}

When approximating the radiative density function by a $\delta$ distribution localised at $X=$ $X_{R}, w(X)$ is then a step function, which justifies the previous approximations in terms of a step function for narrow radiative cooling rates. The trajectory of the solution of the Hamiltonian system eq.( 15) can then be determined analytically. In the hot divertor and cold SOL regimes, the heat flux $Y$ is constant so that $X_{u p}=X_{d i v}+Y_{u p}$ and $X_{d i v}=X_{*}$. We thus compute the solution in the case: $X_{d i v} \leq X_{R} \leq X_{u p}$ such that $Y=Y_{d i v}$ for $X \leq X_{R}$ and $Y=Y_{u p}$ for $X>X_{R}$. The radiation front is then located at $z_{R}$ such that:

$$
\begin{aligned}
z_{R} & =\frac{X_{R}-X_{d i v}}{Y_{d i v}}=1-\frac{X_{u p}-X_{R}}{Y_{u p}} \\
X_{d i v}^{2 / 7} & =X_{*}^{2 / 7}-2 \mathcal{C}_{z}=X_{*}^{2 / 7}\left(1-\frac{2 \mathcal{C}_{z} \mathcal{C}_{\pi}^{2}}{Y_{u p}^{2}}\right) \\
Y_{d i v}^{2} & =Y_{u p}^{2}-2 \mathcal{C}_{z} \mathcal{C}_{\pi}^{2}=Y_{u p}^{2}\left(1-\frac{2 \mathcal{C}_{z} \mathcal{C}_{\pi}^{2}}{Y_{u p}^{2}}\right)
\end{aligned}
$$

Given $U_{d i v}=X_{d i v}^{2 / 7}, U_{R}=X_{R}^{2 / 7}$ and $U_{*}=X_{*}^{2 / 7}$, and the Bohm boundary relation between $Y_{d i v}$ and $U_{d i v}$, one can rewrite this set of conditions in a more convenient way to analyse the location of the radiating front.

$$
\begin{aligned}
z_{R} \mathcal{C}_{\pi} & =\frac{U_{R}^{7 / 2}-U_{d i v}^{7 / 2}}{U_{d i v}^{1 / 2}} \\
U_{*} & =U_{d i v}+2 \mathcal{C}_{z}
\end{aligned}
$$

The radiative front location $z_{R}$ is then determined in terms of $U_{d i v}$ and the control parameter $\mathcal{C}_{\pi}$. One finds that the larger the plasma pressure given by $\mathcal{C}_{\pi}$, the smaller the radiative front distance from the target plate. Furthermore, one can locate $z_{R}=0$ with the equivalent condition $U_{\text {div }}=U_{R}$. The other limit $z_{R}=1$ is set by the curve $\mathcal{C}_{\pi}=\left(1-U_{\text {div }}^{7 / 2}\right) / U_{\text {div }}^{1 / 2}$ in the plane $\left(U_{d i v}, \mathcal{C}_{\pi}\right)$, Fig. 5 left hand side. The operating window for the radiative divertor lies therefore between these two curves, Fig. 5 left hand side. The system leaves the hot divertor regime into the radiative regime when $z_{R}$ becomes positive. The optimum point is set by $z_{R} \approx z_{d}$, when the radiative front has moved away from the target plates to ensures enhanced spreading of radiation onto the plasma facing components, but still lies within the divertor volume. On Fig. 5 left hand side, the region of interest for $z_{d} \approx 0.3$ is thus the transition region in yellow contour plots. When increasing the plasma pressure, and maintaining the radiative front at the same location, one finds a near linear decrease of $U_{d i v}$ (yellow contour lines on Fig. 5 left hand side). This trend in the decrease of the divertor temperature holds down to $U_{\text {div }} \approx 0.2$, Fig. 5 right hand side. The region with negative values of $z_{R}$, in red, corresponds to the hot divertor regime, while the light blue contours set the other limit $z_{R}=1$, namely the cold SOL limit. In fact the cold SOL regime, with the radiation front moving out of the divertor volume into the main chamber SOL corresponds to the green contour plots.

Let us now consider operation at given plasma pressure $\mathcal{C}_{\pi}$, Fig. 5 right hand side. For small values of $\mathcal{C}_{\pi}$ as one decreases $U_{\text {div }}$ one finds that the radiative fronts moves towards the up-stream in a regular fashion. At larger values of the plasma pressure, one finds two 
stages, as $U_{d i v}$ is decreased, the radiative front first shifts linearly with $U_{\text {div }}$ towards the upstream, the displacement rate being smaller when one increases the plasma pressure $\mathcal{C}_{\pi}$. When $U_{d i v}$ approaches zero, the displacement of the front position switches to a rapid up-shift to the upstream for very small changes of $U_{d i v}$, as readily expected from the dependence in $1 / U_{\text {div }}^{1 / 2}$ eq.( $22 \mathrm{a}$ ), Fig. 5 right hand side. Alternatively, assuming that $z_{R}$ is controlled at $z_{d}$, one finds that one must increase the plasma pressure $\mathcal{C}_{\pi}$ to decrease the divertor temperature $U_{d i v}$, small values of $z_{d}$ requiring a large increase in plasma pressure to reduce $U_{d i v}$.

A last step when investigating the properties of the radiative front position is the link between $U_{d i v}$ and $U_{*}$ the actual control parameter. From eq.( $\left.22 \mathrm{~b}\right)$, one finds that the behaviour with respect to decreasing the input power $U_{*}$ is the same as reducing $U_{d i v}$ except that $U_{*}$ is up-shifted by $2 \mathcal{C}_{z}$ which corresponds to the radiative loss that occurs at $z_{R}$. Given the definition $Y_{u p}=\mathcal{C}_{\pi} U_{*}^{1 / 2}$, one can then close the calculation since the radiative front properties, the front location $z_{R}$, the divertor temperature $U_{\text {div }}$ and energy outflux $Y_{d i v}$ can now be determined by the three control parameters: $Y_{u p}$, the input energy flux on the field line, $\mathcal{C}_{\pi}$, the plasma pressure and $\mathcal{C}_{z}$ the impurity concentration, Fig. 6. One can readily see in the contour plot, left hand side of Fig. 6 , and variation of $Y_{\text {up }}$ versus $U_{\text {div }}$, Fig. 6 right hand side, that in the radiative region, higher energy influx is required to sustain comparable divertor conditions, which is fully consistent with the idea of energy dissipation by radiation. Furthermore, at given $Y_{u p}$, there is a region such that three values of $U_{d i v}$ can be achieved, consistently with the discussion on thermal bifurcations Section 3.2.

\subsection{Constraint on the upstream temperature}

With respect to prior work, the present analysis also allows one determining the upstream plasma temperature which governs the occurrence of the cold SOL regime. The expression of $U_{u p}$ is readily computed from eq. (21a) and can be compared to $U_{u p}^{*}$ the thermal energy that would be achieved under the same conditions, hence with the same control parameters
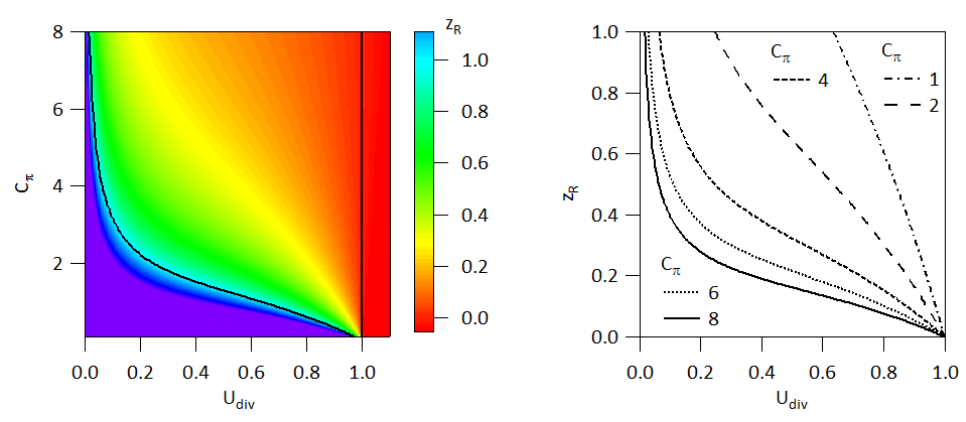

Figure 5: Front location $z_{R}$ in the plane $\left(U_{d i v}, \mathcal{C}_{\pi}\right)$, left hand side. The black curves indicate the limit regimes: hot divertor regime $z_{R}=0$, vertical line, and the cold SOL regime $z_{R}=1$. Position of the radiative front versus the divertor temperature $U_{\text {div }}$ for different values of the plasma pressure $\mathcal{C}_{\pi}$, right hand side. 

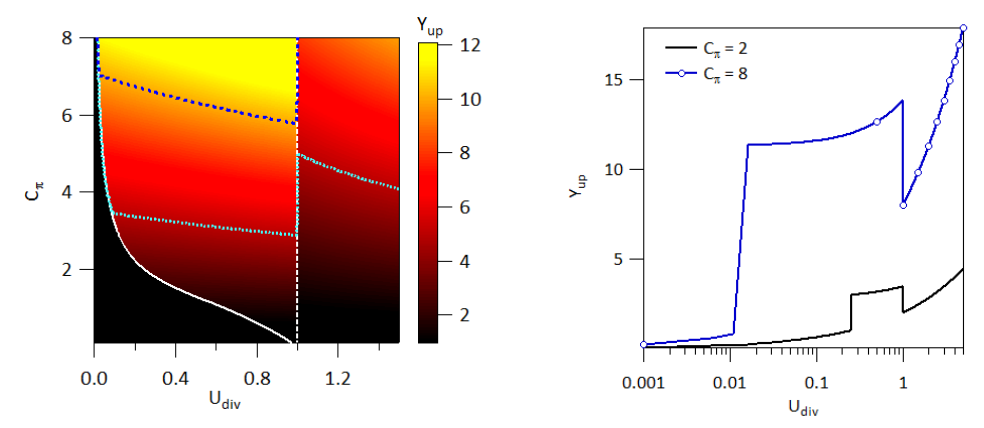

Figure 6: Upstream energy influx $Y_{u p}$ in the plane $\left(U_{d i v}, \mathcal{C}_{\pi}\right)$, left hand side. The white contours identify the radiative divertor region and the dashed lines iso- $Y_{u p}$ lines, dotted (cyan) line for $Y_{u p}=5$ and dashed (blue) line for $Y_{u p}=10$. At given plasma pressure, $\mathcal{C}_{\pi}$, one can also determine the upstream heat flux variation $Y_{u p}$ corresponding to given divertor temperature $U_{d i v}$, right hand side.

$Y_{u p}, \mathcal{C}_{\pi}$, but without radiation, Fig. 7

$$
\begin{aligned}
& X_{u p}=Y_{u p}\left(1-z_{R}\right)+Y_{d i v} z_{R}+X_{d i v} \\
& X_{u p}^{*}=Y_{u p}+X_{*}
\end{aligned}
$$

One thus finds that $U_{u p} \leq U_{u p}^{*}$ since the difference $X_{u p}^{*}-X_{u p}=\left(Y_{u p}-Y_{d i v}\right) z_{R}+X_{*}-X_{d i v}$ is the sum of two positive contributions, the former induced by the difference in temperature gradient stemming from the transport properties, and the latter due to the difference in divertor temperatures, governed by eq.( $22 \mathrm{~b})$. This variation in upstream values of $X$ tends to increase both with the distance from the radiative front to the divertor $z_{R}$, and with the heat flux drop at the radiative front $Y_{u p}-Y_{d i v}$. A more convenient expression can be computed using eq.( 22):

$$
X_{u p}^{*}-X_{u p}=z_{R} \mathcal{C}_{\pi} \frac{Y_{u p}}{\mathcal{C}_{\pi}}\left(1-\frac{Y_{\text {div }}}{Y_{u p}}\right)+X_{*}\left(1-\frac{X_{d i v}}{X_{*}}\right)
$$

These various contribution can then be readily be rewritten in terms of $U_{*}$ standing for the heat flux source and the ratio $U_{d i v} / U_{*}$ which characterises the radiative divertor performance, Fig. 7 left hand side. Not all points in this plot are consistent with the assumptions. In particular negative values of $z_{R}$, which correspond to the hot divertor regime above the plain line, are such that $X_{u p}^{*}-X_{u p}=0$. The other limit regime of the cold SOL regime $z_{R}=1$ are also characterised by $X_{u p}^{*}-X_{u p}=0$. The location of this transition depends on the plasma pressure. The dashed line on Fig. 7 left hand side corresponds to $z_{R} \mathcal{C}_{\pi}=1$ and stands for this second transition provided $\mathcal{C}_{\pi}=1$. Note that in this form, the drop in $X$ is normalised by $X=1$, namely the value at the peak of the cooling rate at thermal energy $T_{R}$. The variation by a factor one, the yellow contour line at zero value in log scale, can therefore be large. The variation of $X$ takes the form of a step function. The large values located towards the top left hand side correspond to the high performance divertor operation at large values of $U_{*}$ and small values of $U_{\text {div }} / U_{*}$. However, one finds that these values are located in the region with no solution, below the plain curve with open circle markers. This property is recovered when considering the temperature difference $U_{u p}-U_{u p}^{*}$, Fig. 8. In this plot the constraint on the radiative 

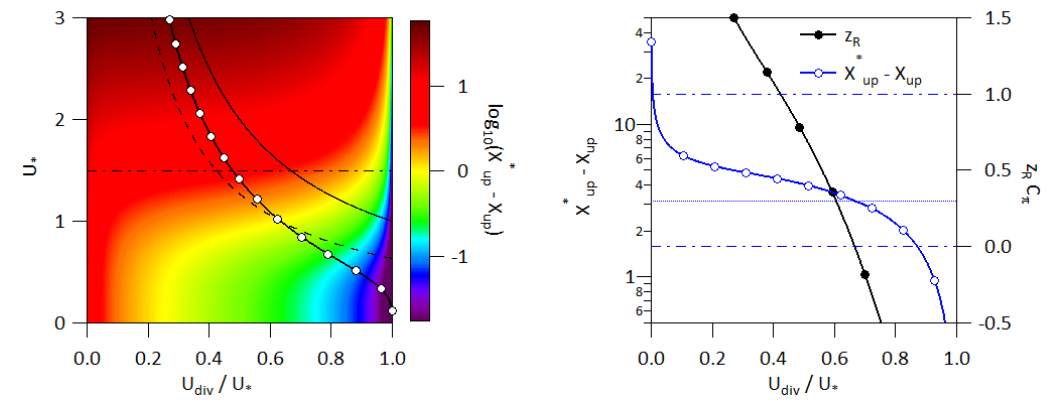

Figure 7: Variation of the upstream temperature $X_{u p}^{*}$ in the case without radiation and $X_{u p}$ with radiation. Left hand side, contour plots of the difference $X_{u p}^{*}-X_{u p}$ in $\log$ scale in the parameter space $U_{\text {div }} / U_{*}$ giving the divertor performance and $U_{*}$ standing for the input power. The plane line determines the transition from the hot divertor to the radiative divertor regimes. The dashed line stands at the transition from the radiative to the cold SOL regimes for $\mathcal{C}_{\pi}=1$. The plain line open circles is the limit of radiative divertor solutions, which corresponds to the transition to zero solutions. The dash-dot line corresponds to the chosen value of $U_{*}$ used to plot the profile, right-hand side, $X_{u p}^{*}-X_{u p}$ versus $U_{d i v} / U_{*}$, plain line, open circles. The variation of the location of the radiative front given by $z_{R} \mathcal{C}_{\pi}$ versus $U_{d i v} / U_{*}$ is given by the line with closed circles markers, right hand side scale. The dashed dot line at the transition points into the radiative regime and the plain horizontal line is $z_{d}=0.3$ for $\mathcal{C}_{\pi}=1$.

regime is clearly visible with loss of solutions at small values of $U_{\text {div }} / U_{*}$ for all values of $U_{*}$, Fig. 8 left hand side, as well as small values of $U_{*}$, Fig. 8 right hand side. The range of values for the radiative divertor determined by the location of the radiative front yields a narrow range of values for the drop in upstream temperature with $U_{u p}^{*}-U_{u p} \approx 0.5$ which means that the upstream plasma is changed by the radiative divertor operation. Should this temperature drop be compensated by a density increase to maintain the same upstream pressure would then lead to an enhancement of upstream plasma collisionality and consequent impact on confinement properties.

\subsection{Input power ramp-up at given radiative front position}

As discussed is Section 4.1, radiation must be localised in the divertor volume, $z \leq z_{d}$ and at a distance from the target plates $(z=0)$ to minimise the radiative heat deposition on the target plates, hence $z_{R} \approx z_{d}$, Fig. 1 . It is then interesting to set as control condition a prescribed value of $z_{R}$, here $z_{R}=0.3$. Then given eq.(22a), one finds that either the plasma pressure $\mathcal{C}_{\pi}$ or the divertor temperature $U_{d i v}$ are prescribed. Since we are interested in lowering the divertor temperature and energy flux, we assume that the controlled position of the radiative front defines $\mathcal{C}_{\pi}$ in terms of $U_{\text {div }}$, Fig. 9 left hand side. One then finds that the limit $\mathcal{C}_{\pi} \rightarrow 0$ corresponds to the onset of the radiative divertor regime. Negative values that are meaningless regarding the physics then correspond to the hot divertor regime, without radiation. Conversely, the limit $U_{d i v} \rightarrow 0$ then requires very large plasma pressure to be achieved $\mathcal{C}_{\pi} \rightarrow+\infty$. In order to define $U_{*}$ we consider $\mathcal{C}_{z}=0.4$, hence given $\mathcal{C}_{\pi}$, the upstream heat flux $Y_{u p}$ is determined for each value of the divertor temperature $U_{\text {div }}$, Fig. 9 right hand side. 

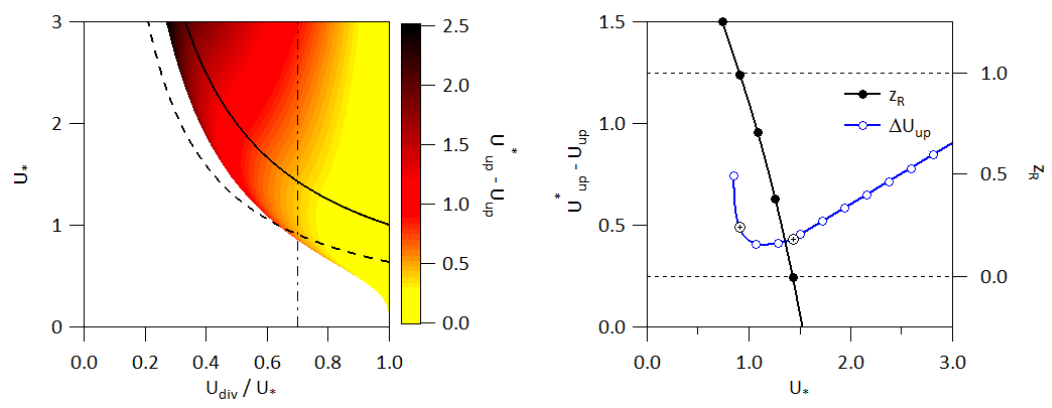

Figure 8: Variation of the upstream temperature $U_{u p}^{*}$ in the case without radiation and $U_{u p}$ with radiation. Left hand side, contour plots of the difference $U_{u p}^{*}-U_{u p}$, linear scale, in terms of $U_{d i v} / U_{*}$ and $U_{*}$. The transition from the hot divertor to the radiative divertor regimes is given by the plain line. The dashed line stands at the transition from the radiative to the cold SOL regimes for $\mathcal{C}_{\pi}=1$. The dash-dot line corresponds to the chosen value of $U_{d i v} / U_{*}$ used to plot the profile, right-hand side, $U_{u p}^{*}-U_{u p}$ versus $U_{*}$, plain line, open circles. The profile of $z_{R} \mathcal{C}_{\pi}$ versus $U_{*}$ is given by the line with closed circles, right hand side scale. The dashed dot line at the transition points into the radiative regime for $\mathcal{C}_{\pi}=1$.

This result then yields the system response during a ramp-up of the injected heat flux $Y_{u p}$ for given $\mathcal{C}_{z}$ and optimised location of the radiative front. When $Y_{u p}$ is small, one finds that the divertor temperature is comparable to that of the radiative front $U_{\text {div }} \leq U_{R}=1$ and $Y_{\text {div }} \leq Y_{u p}$ with similar behaviour. One also finds that $U_{*} \geq U_{d i v}$, the divertor temperature without radiation with otherwise same conditions, is also approximatively constant and not very different from $U_{d i v}$, Fig. 9 right hand side. At $Y_{u p} \approx 5$, a change of regime is observed with a steady decrease of the divertor temperature $U_{d i v}$ when increasing $Y_{u p}$ while the heat flux to the divertor levels-off, Fig. 9 right hand side. In this second regime, radiation is effective in lowering the divertor temperature and in decoupling the divertor heat flux from the upstream heat flux. This regime is obtained by monitoring the plasma pressure as shown on Figure 9 left hand side.

To complete this analysis, let us investigate the case of prescribed $\mathcal{C}_{\pi}$ and therefore varying the radiative front location $z_{R}$, Fig. 10 left hand side. Negative values of $z_{R}$ correspond to the hot divertor regime. At $z_{R}=0$ the system enters the radiative regime which also corresponds to $U_{d i v}=U_{R}=1$. For $z_{R}=1$ the system has switched into the cold SOL regime as $U_{u p}=U_{R}=1$. On Fig. 10 left hand side, one can readily notice the increase of the input energy flux that is required to sustain the radiative regime at gradually increasing position of the radiative front and decreasing divertor temperature. It is also to be underlined that the upstream temperature weakly increases while $U_{*}$ increases by a factor 2 as $U_{d i v}$ decreases below $U_{R}=1$.

\subsection{Radiative efficiency}

Properties of the radiative divertor thus depend both on independent control parameters, namely $Y_{u p}, \mathcal{C}_{\pi}$ and $\mathcal{C}_{z}$, and on important criteria for divertor operation such as the location 

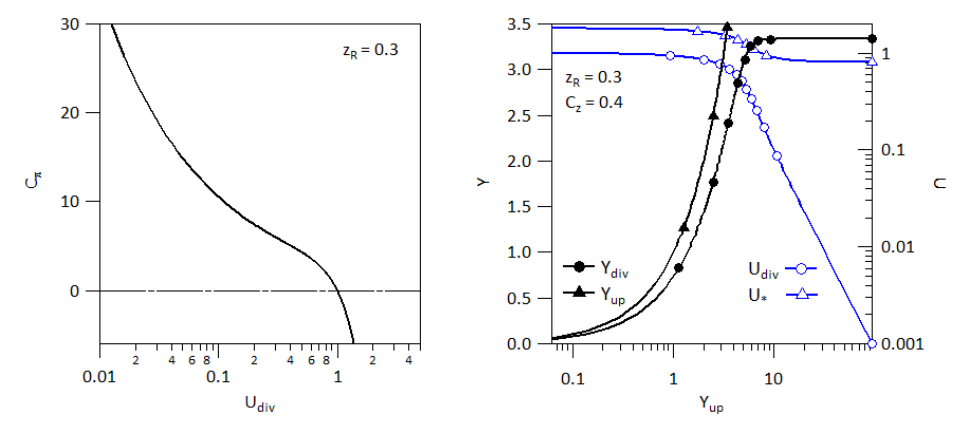

Figure 9: For a given front location $z_{R}=0.3$, required plasma pressure $\mathcal{C}_{\pi}$ versus $U_{\text {div }}$, left hand side. On the right hand side, ramp-up of the input heat flux $Y_{u p}$ with fixed radiative front location $z_{R}=0.3$ and prescribed impurity seeding, $\mathcal{C}_{z}=0.4$. The heat flux onto the divertor $Y_{d i v}$ closely matches $Y_{u p}$, and then levels off, right hand side scale. Conversely, the divertor temperature $U_{d i v}$ is first approximately constant and then drops steadily, left hand side scale, while $U_{*}$ exhibits a step down that takes into account the change in plasma pressure $\mathcal{C}_{\pi}$ required to sustain the operation constraints, left hand side.

of the radiative front Section 4.1. Of interest is also the temperature drop along the field line, $U_{u p}-U_{d i v}$, Fig. 11 left hand side, and the heat flux drop along the field line $Y_{u p}-Y_{d i v}$ Fig. 11 right hand side. For both criteria, one finds that the largest drop along the field line takes place at the limit $U_{d i v} \rightarrow 0$ towards the cold SOL regime. For a prescribed location of the radiative front, for example at $z_{R}=0.3$, dashed line on Fig. 11, one finds that the change in heat flux $Y_{u p}-Y_{\text {div }}$ first steps up when entering the radiative regime but really increases when the divertor temperature becomes small, which, following the line $z_{R}=0.3$, only occurs at highest plasma pressure. A trade-off between various aspects of the divertor physics is thus required to evaluate the radiative divertor efficiency.

Let us simplify the discussion by introducing the efficiencies $\varepsilon_{U}=1-U_{d i v} / U_{*}$ and $\varepsilon_{Y}=$ $1-Y_{\text {div }} / Y_{u p}$, hence the gain achieved on the divertor temperature with radiation compared to that obtained without, $\mathcal{C}_{z}=0$. One finds that $\varepsilon_{U}$ depends on a single combination of the control parameters and that $\varepsilon_{Y}$ can be expressed in terms of $\varepsilon_{U}$, Fig. 10 right hand side.

$$
\begin{aligned}
& \varepsilon_{U}=\frac{U_{*}-U_{d i v}}{U_{*}}=\frac{2 \mathcal{C}_{z} \mathcal{C}_{\pi}^{2}}{Y_{u p}^{2}}=\frac{2 \mathcal{C}_{z}}{U_{*}} \\
& \varepsilon_{Y}=\frac{Y_{u p}-Y_{d i v}}{Y_{u p}}=1-\sqrt{\frac{U_{d i v}}{U_{*}}}=1-\sqrt{1-\varepsilon_{U}}
\end{aligned}
$$

These efficiencies thus depend on a single combination of the control parameters, namely $2 \mathcal{C}_{z} / U_{*}$. This parameter can be read as the ratio of the cooling capability, characterised by $2 \mathcal{C}_{z}$, and the input heat flux, characterised by $U_{*}$. Given the constraint $\varepsilon_{U} \leq 1$, one recovers a constraint on $U_{*}, U_{*} \geq 2 \mathcal{C}_{z}$. It is to be underlined that the optimum efficiency $\varepsilon_{U}=1$ is achieved at the limit $U_{d i v}=0, Y_{d i v}=0$ a singular operation point that belongs to the cold SOL regime. 

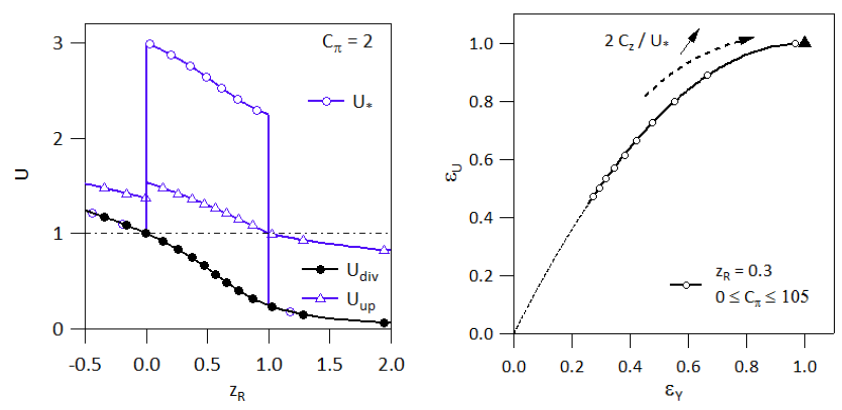

Figure 10: Left hand side, temperature variation versus $z_{R}$ at given plasma pressure $\mathcal{C}_{\pi}=2$, closed circle markers $U_{\text {div }}$, upstream temperature $U_{u p}$ open triangles, upstream influx characterised by $U_{*}$, open circles. Right hand side, radiative efficiency in terms of divertor temperature lowering, $\varepsilon_{U}$ versus the radiative efficiency in terms of lowering the heat flux, $\varepsilon_{Y}$. The arrow indicated the trend towards enhance performance when the control parameter $2 \mathcal{C}_{z} / U_{*}$ is increased.
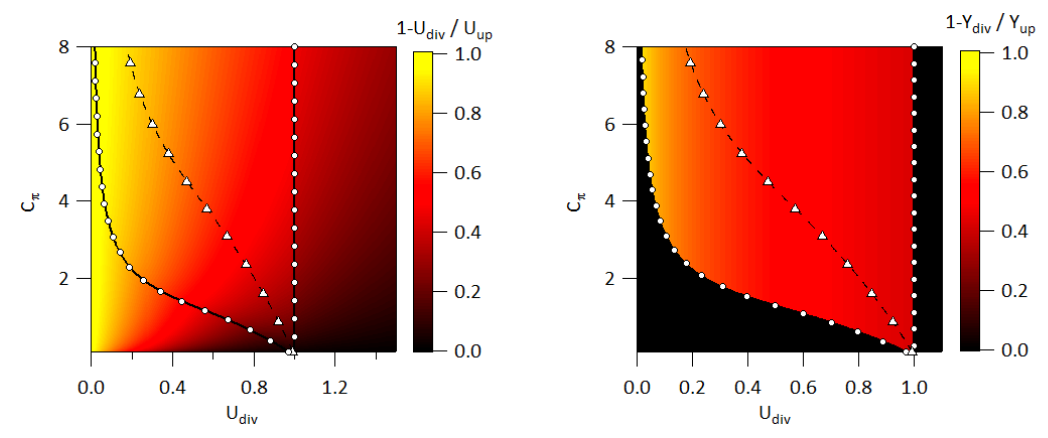

Figure 11: Divertor performance, left hand side $\left(U_{u p}-U_{\text {div }}\right) / U_{u p}$, right hand side $\left(Y_{u p}-\right.$ $\left.Y_{d i v}\right) / Y_{u p}$ in the parameter space $\mathcal{C}_{\pi}$ versus $U_{\text {div }}$. The radiative divertor operation is bounded by the plain lines open circles, the vertical line at $U_{d i v}=1$ for $z_{R}=0$ and the other line towards lower values $U_{d i v}$ for $z_{R}=1$. The dashed line with open triangles locates the position of the radiative front at $z_{R}=0.3$.

\subsection{Constrained operation due to the radiative bifurcations}

As addressed in Section 3.2, when the step of the radiative work is sharp enough due to a strong peaking of the radiation density function, one finds multiple solutions for a given energy flux driving the SOL and divertor system. In the case of a radiation density function defined by a Dirac distribution, the bifurcation appears as a jump and the region with negative slope is of zero extent, Fig. 12. As discussed previously the solution presents 3 branches, a hot divertor branch such that $U_{d i v}=U_{*}$ that extends from the large values of $U_{*}$ down to $U_{*}=1$ when the system enters the radiative divertor regime. In the radiative divertor regime one has $U_{d i v}=U_{*}-2 \mathcal{C}_{z}$ and $U_{u p} \geq 1$. The radiative divertor regime is therefore such that it coexists with the hot divertor regime since for a given value of $U_{*} \geq 2 \mathcal{C}_{z}$, one can find two values for $U_{d i v}$, namely $U_{d i v}=U_{*}$ for the hot divertor regime and $U_{d i v}=U_{*}-2 \mathcal{C}_{z}$ in the radiative divertor regime. The radiative divertor regime is sustained until $U_{u p}=1$, hence for $U_{*}$ equal to $V_{*}$ such that 
$1=\mathcal{C}_{\pi} V_{*}^{1 / 2}+V_{*}^{7 / 2}$. For large values of $\mathcal{C}_{\pi}^{2}$, one thus finds that $V_{*}$ scales like $V_{*} \approx \mathcal{C}_{\pi}^{-2}$. For $U_{*} \leq V_{*}$, the only branch is the cold SOL solution. The radiative solution is therefore found for $V_{*}+2 \mathcal{C}_{z} \leq U_{*} \leq 1+2 \mathcal{C}_{z}$.

When decreasing the value of $U_{*}$, the system is first in the hot divertor regime. When $U_{*}=1$ is reached this solution does not exist any more. The system cannot switch directly to the cold SOL regime since $V_{*}<1$. It must then go into the radiative regime which is only possible if $V_{*}+2 \mathcal{C}_{z} \leq 1 \leq 1+2 \mathcal{C}_{z}$, hence the trivial condition $\mathcal{C}_{z}>0$ and the condition $V_{*} \leq 1-2 \mathcal{C}_{z}$. This yields the necessary condition $\mathcal{C}_{z} \leq 0.5$. When the condition cannot be satisfied as on Fig. 12, the ramp-down of the input power does not allow one to reach the radiative divertor regime since no solution is found for $U_{*}$ just under 1 . Similarly, the ramp-up of $U_{*}$ starting from the cold SOL regime, also leads to a loss of the solution when $U_{*}$ increases beyond $V_{*}$ because the radiation exceeds the available power. Considering a constraint on the value of $z_{R}$, one then finds that reaching the radiative regime with a ramp of the heating power is difficult. One must first decrease the heating power to switch into the radiating regime, however with the radiative front ahead of the divertor volume, in the SOL region, and then increase the heating power to monitor the radiative front into the divertor volume.

For $\mathcal{C}_{\pi}=8$, Fig. 12 right hand side, one finds that the cold SOL regime reached when $U_{u p} \leq 1$, see curve with open square markers (blue), only exists in a very narrow range of small $U_{*}$ values. In this case, the operation of the cold SOL regime can be expected to be difficult to control.

\subsection{Density regimes with radiation}

In Section 4.5 we have addressed the bifurcation aspect by considering a ramp of the input power. When addressing the divertor density regimes, one introduces the upstream density $n_{u p}$ as control parameter. We then have in mind that this density acts as a boundary condition for the core density, and consequently that for each value of the core density one defines a unique value of $n_{u p}$ proportional to the core density. The standard experimental procedure of a core density ramp then translates into a ramp of $n_{u p}$ for the present model. Since $\Pi_{u p}=n_{u p}\left(T_{u p}^{e}+T_{u p}^{i}\right)$, one defines the density normalisation as $n_{T}=$
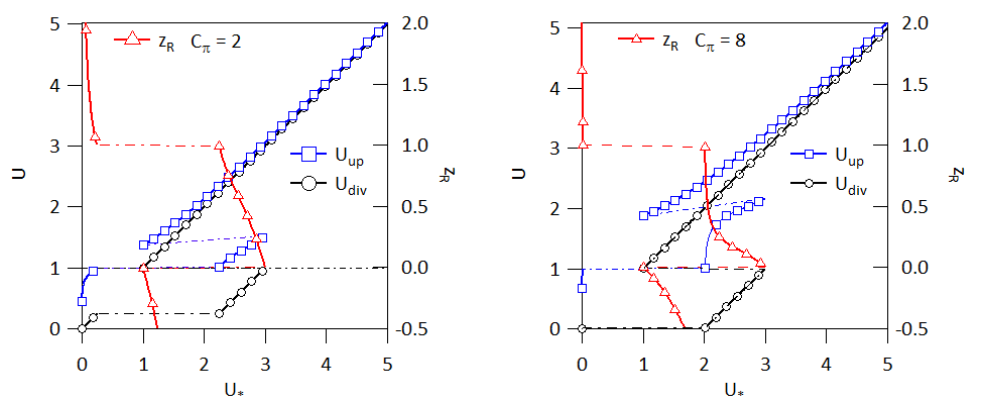

Figure 12: Upstream, $U_{u p}$ open square markers, and divertor, $U_{\text {div }}$ open circle markers, temperatures versus $U_{*}$, left hand side scale and location of the radiative front $z_{R}$, open triangles, versus $U_{*}$, right hand side scale for $\mathcal{C}_{z}=1$ and two values of the plasma pressure control parameter $\mathcal{C}_{\pi}, \mathcal{C}_{\pi}=2$ left hand side figure, and $\mathcal{C}_{\pi}=8$, right hand side figure. 
$\Pi_{T} /\left(2 T_{R}\right)$, hence the normalised density $N=n / n_{T}$. One then obtains the straightforward expression for $N_{u p}$ in terms of the control parameter $\mathcal{C}_{\pi}$ and of the upstream temperature $U_{u p}$ that depends on the computed solution.

$$
N_{u p}=\frac{\mathcal{C}_{\pi}}{U_{u p}}
$$

Let us first recover the density regimes without radiative losses, therefore $Y_{d i v}=Y_{u p}$ and $X_{u p}=Y_{u p}+X_{d i v}$ so that:

$$
N_{u p}=\frac{Y_{u p} / U_{d i v}^{1 / 2}}{\left(Y_{u p}+X_{d i v}\right)^{2 / 7}}
$$

Taking into consideration the pressure balance $\Pi_{u p}=\Pi_{d i v}$ then also yields $N_{d i v}=$ $N_{u p} U_{u p} /\left(2 U_{\text {div }}\right)$ and therefore the divertor density.

$$
N_{d i v}^{1 / 3}\left(\left(\frac{Y_{u p}}{4 N_{d i v}}\right)^{7 / 3}+Y_{u p}\right)^{-2 / 7}=2^{1 / 3} N_{u p} Y_{u p}^{-2 / 3}
$$

By considering asymptotic limits in (27), one recovers two branches of the divertor density regime: The first case $X_{d i v} \gg Y_{u p}$ corresponds to the linear regime, when the divertor density responds linearly to the core density increase. This regime is also called sheath limited regime. The second $X_{d i v} \ll Y_{u p}$ is the high recycling regime. In the former case one finds that $U_{u p} \approx U_{d i v}$ while in the latter $U_{u p} \approx Y_{u p}^{2 / 7}$, the upstream temperature condition is found to be independent from the divertor solution. Scaling laws for the various divertor and upstream characteristic parameters in terms of the control parameters are then readily obtained:

$$
\begin{array}{ccc}
\text { linear regime } & ; & \text { high recycling regime } \\
X_{d i v} \gg Y_{u p} & ; & X_{d i v} \ll Y_{u p} \\
U_{d i v} \approx Y_{u p}^{2 / 3} N_{u p}^{-2 / 3} & ; & U_{d i v} \approx Y_{u p}^{10 / 7} N_{u p}^{-2} \\
U_{u p} \approx Y_{u p}^{2 / 3} N_{u p}^{-2 / 3} & ; & U_{u p} \approx Y_{u p}^{2 / 7} \\
N_{d i v} \approx \frac{1}{2} N_{u p} & ; & N_{d i v} \approx 2 Y_{u p}^{-8 / 7} N_{u p}^{3} \\
\Gamma_{d i v} \approx \frac{1}{2} N_{u p}^{2 / 3} Y_{u p}^{1 / 3} & ; & \Gamma_{d i v} \approx 2 Y_{u p}^{-3 / 7} N_{u p}^{2} \\
\Pi \approx Y_{u p}^{2 / 3} N_{u p}^{1 / 3} & ; & \Pi \approx Y_{u p}^{2 / 7} N_{u p}
\end{array}
$$

The framework of divertor regimes is operating at given input power hence fixed value of $Y_{u p}$ and ramping the density, usually up from the hot into the high recycling divertor regime. In the standard log-log plots to highlight the scaling laws, changing the input power leads to i) a horizontal shift of the critical density to switch from one regime to the other since $N_{u p}^{\text {crit }} \approx \sqrt{2} Y_{u p}^{4 / 7}$, ii) a vertical shift given the dependence on $Y_{u p}$. It is to be noted that the transition between the two regimes, $X_{d i v} \approx Y_{u p}$ does not depend on the particular normalisation $T_{R}$ that is chosen for the temperature, indeed it leads to:

$$
\left(\frac{T_{d i v}}{T_{R}}\right)^{7 / 2}=X_{d i v} \approx Y_{u p}=\frac{Q_{u p}}{Q_{T}}=\frac{Q_{u p} L_{\|}}{\frac{2}{7} \kappa_{0}^{e}}\left(\frac{1}{T_{R}}\right)^{7 / 2}
$$


However, the way radiation modifies these standard divertor regimes depends on both $Y_{u p}$ and $T_{R}$, the temperature at the peak of the radiative density function. At low input power or large $T_{R}$, so that $Y_{u p} \ll 1$, the condition $X_{\text {div }} \gg Y_{u p}$ can occur for $X_{\text {div }} \leq 1$. Radiation will then modify the linear regime. Conversely, for $Y_{u p} \geq 1$, hence at higher input power or lower radiative temperature $T_{R}$, one finds that radiation modifies the high recycling regime. The changes governed by radiation are the possible occurrence of bifurcations and consequently loss of solutions together with replacing the scaling laws by more complex dependences, as can be noticed on Fig. 13 left hand side.

For the case without radiation, open circles, one recovers the scaling laws eq.( 29b). With impurity seeding at given concentration, the hot divertor regime is not modified, red curve closed triangles. At $N_{u p}^{c r i t} \approx 2$ and increasing density, there is a transition to the radiative regime, black curve closed circles. In the latter regime, $U_{u p}$ decreases to 1 , which corresponds therefore to the cold SOL regime, and there is no solution at density larger than $N_{u p}^{c r i t} \approx 4$.4. One finds therefore a radiative limit for the density. Note that this limit is not the so-called Greenwald limit[6] since the latter is defined for operation with little radiation and must be understood therefore as an upper limit at vanishing line radiation.

One thus finds that constraints exist in the control parameter space to obtain or not solutions. These depend on the way control parameters are varied. In particular, the response to a scan in $Y_{u p}$ is different from that in $N_{u p}$. The effect of the control parameters is also exemplified on Fig. 13 right hand side. The lines draw the boundaries of the region with $0 \leq z_{R} \leq 1$ in the space of control parameters $\left(Y_{d i v}, \mathcal{C}_{z}\right)$ for two given values of $Y_{u p}$. The plain line $z_{R}=0$, that marks the transition from the hot divertor, $U_{\text {div }}>1$, hence $z_{R}<0$ does not depend on the input energy flux $Y_{u p}$. In contrast the line $z_{R}=1$, the transition to the cold SOL regime exhibits a marked dependence on $Y_{u p}$. At low energy flux input $Y_{u p}=0.75$, closed circles, there is only a narrow operating region for the radiative regime between $z_{R}=0$ and $z_{R}=1$. At larger input power, $Y_{u p}=3.00$, the parameter space opens up and only narrows again towards the larger impurity concentration and lower energy flux to the divertor, Fig. 13 right hand side.

The transition to zero solutions at high upstream density can be understood when considering the location of the radiating front $z_{R}$ Fig. 14, left hand side. Ramping up the density, a transition occurs at $N_{u p}^{\text {crit }} \approx 2$ from the hot divertor values $z_{R} \leq 0$ to the radiative range of values $0 \leq z_{R} \leq 1$, the upper $z_{R}=1$ value being reached at $N_{u p}^{\text {crit }} \approx 4.4$. The transition to the cold SOL regime is then not possible because the upper limit of $z_{R}$ in this regime is $1 / Y_{u p}$, which is smaller that 1 , hence in the radiating regime for the chosen value of $Y_{u p}=3$, Fig. 14, left hand side.

The core plasma density ramp akin to the upstream density increase is a standard experimental procedure in present experiments but is only relevant for the operation of burning plasmas when one considers density limit issues. Regarding the fusion power performance the key parameter is the core plasma pressure that can also be assumed to scale linearly with its boundary value $C_{\pi}$. An example of divertor operation in terms of plasma pressure is given on Fig. 14, right hand side. One then finds that there is no distinction between the hot divertor and high recycling regimes since $N_{d i v}$ scales like $C_{\pi}^{3}$ in both regimes, see eq.( 29c) and eq.( 29e). Impurity seeding adapted to radiation within the divertor volume $z_{R}<1$ then introduces a radiative limit to the plasma pressure char- 
acterised by a faster drop of the plasma temperature and consequently a faster increase of the divertor density. This issue that remains to be addressed is then to determine if introducing these radiative limits, either on the plasma density or plasma pressure, are balanced by an improved divertor operation point.

Two figures of merit are then to be considered, the drop in plasma electron temperature, Fig. 15 left hand side, and in heat flux to the divertor Fig. 15 right hand side. Regarding the latter, one finds that in the radiative regime the heat flux drops from $\approx 83 \%$ to $\approx 33 \%$ of the heat flux without radiation while the divertor electron temperature drops by a factor that reaches 5 compared to the case at the same upstream density without radiation, Fig. 15 left hand side. This factor can compensate the effect of enhanced sputtering due to the larger charge of the ionised impurities impinging onto the target plate. In order to remain below the sputtering threshold assuming that this value is marginally achieved with deuterium in the case without impurity seeding, the reduced divertor electron temperature then extends the divertor operation domain provided the seeded impurity has a charge $Z \approx 5$, therefore not appropriate for neon $Z=10$, the first noble gas (hence with no deleterious chemical reactivity) above helium.

\subsection{Constrained location of the radiative front in the divertor density regimes}

Divertor operation with density or plasma pressure ramp-up and impurity seeding, Section 4.6, is characterised by a rather marginal improvement of the divertor operation window since the drop in heat flux to the target plate while being significant does not appear to be backed by a sufficient drop in the divertor plasma temperature $U_{\text {div }}$ to alleviate the issue of enhanced sputtering by the seeded impurity. Moreover, these results are obtained with no constraint on the location of the radiative front. In fact, one can readily show that a constraint on the radiative front location leads to a conflict with the goal of reducing the energy flux to the target plate since one must maintain a minimum energy flux to sustain the temperature gradient between the radiative front with $U_{R}=1$ and the divertor with $U_{\text {div }} \rightarrow 0$. This property is determined by eq.( $22 \mathrm{a}$ ), which for given $z_{R}$ determines the plasma pressure for a given temperature at the divertor, and consequently the energy flux to the target plate.

$$
Y_{\text {div }}=\mathcal{C}_{\pi} U_{\text {div }}^{1 / 2}=\frac{1}{z_{R}}\left(1-U_{d i v}^{7 / 2}\right)
$$

The largest flux to the divertor is therefore obtained when $U_{\text {div }} \rightarrow 0$ and is then solely determined by the prescribed position of the radiative front. Conversely, a small energy flux is achieved at large divertor temperature, $U_{\text {div }} \rightarrow U_{R}=1$. To complete the determination of the divertor operation point, a prescribed input power $Y_{u p}$ then determines the impurity concentration $\mathcal{C}_{z}$ given the energy balance equation eq.( $\left.21 \mathrm{c}\right)$.

$$
\mathcal{C}_{z}=\frac{Y_{u p}^{2} z_{R}^{2}-\left(1-U_{d i v}^{7 / 2}\right)^{2}}{2\left(1-U_{d i v}^{7 / 2}\right)^{2}} U_{d i v}
$$

Upstream temperature conditions are determined by the location of the radiative front and by the input power since $U_{u p}=\left(Y_{u p}\left(1-z_{R}\right)+1\right)^{2 / 7}$. Regarding the upstream density, 
one then finds that it depends on both: the conditions that prevail in the divertor volume and determine the plasma pressure $\mathcal{C}_{\pi}$, eq.( 31$)$, as well as on upstream conditions, namely the upstream temperature, since $N_{u p}=\mathcal{C}_{\pi} / U_{u p}$. In order to maximise the plasma pressure while avoiding a too large upstream density, one must therefore achieve a high upstream temperature.

In the limit $U_{d i v} \rightarrow 0$, the radiative divertor regime can be controlled in a way to achieve a decoupling between the upstream conditions, with specific control parameter $Y_{u p}$, and the divertor conditions determined by the impurity concentration $\mathcal{C}_{z}$, with $U_{\text {div }} \approx 2 \mathcal{C}_{z} /\left(Y_{u p}^{2} z_{R}^{2}\right)$, since $Y_{u p} z_{R} \gg 1$ is required to ensure both low divertor temperature and low seeding impurity concentration. This yields the plasma pressure $\mathcal{C}_{\pi} \approx$ $Y_{u p} /\left(2 \mathcal{C}_{z}\right)^{1 / 2}$ and upstream temperature, $U_{u p} \approx\left(Y_{u p}\left(1-z_{R}\right)\right)^{2 / 7}$. Consequently, one determines the upstream density $N_{u p} \approx Y_{u p}^{5 / 7} /\left(2 \mathcal{C}_{z}\right)^{1 / 2} /\left(1-z_{R}\right)^{2 / 7}$. It is yo be noted that in this regime $Y_{d i v} \rightarrow 1 / z_{R}$ so that $Y_{u p} \gg Y_{d i v}$. If one now assumes that in this regime the plasma is running close to a density limit $N_{\text {crit }}$, one can prescribe a further condition, $\left(2 \mathcal{C}_{z}\right)^{1 / 2} \geq Y_{u p}^{5 / 7} /\left(N_{c r i t}^{1 / 2}\left(1-z_{R}\right)^{2 / 7}\right)$ in order to prevent overriding it, $N_{\text {up }} \leq N_{\text {crit }}$. This bounds the performance of the radiative divertor by setting an upper limit to the plasma pressure $\mathcal{C}_{\pi} \leq Y_{u p}^{2 / 7}\left(1-z_{R}\right)^{2 / 7} N_{\text {crit }}^{1 / 2}$ and a lower limit to the divertor temperature $U_{\text {div }} \geq 1 /\left(N_{\text {crit }}^{1 / 2} Y_{u p}^{4 / 7} z_{R}^{2}\left(1-z_{R}\right)^{2 / 7}\right)$. It is to be underlined that increasing $Y_{u p}$ and consequently $\mathcal{C}_{z}$ allows one to enhance the divertor performance by increasing the upper bound in plasma pressure and decreasing the lower bound in plasma temperature at the divertor target plate.

\section{Controlled divertor operation}

\subsection{Divertor regimes for different impurity species}

The analysis presented in Section 3 and Section 4 is performed from a mathematical point of view, the underlying physics being taken into account by the various normalisation that have been introduced. For a class of light impurities, one finds that the normalisation of the radiative function is typically $\mathcal{L}_{R} \approx 10^{-30} \mathrm{~W} \mathrm{~m}^{3}$. The normalising heat flux, $Q_{T}$ and pressure $\Pi_{T}$ then depend explicitly on the impurity species via the choice of $T_{R}$ and on the divertor geometry via the length $L_{\|}$. One can use this data to determine $Q_{R} / c_{z}$ and $n_{T}$ which are useful to bridge the mathematical results to experimental data.

$$
\begin{aligned}
Q_{T} & \approx 0.9110^{3} \mathrm{Wm}^{-2} \frac{\left(T_{R} / 1 \mathrm{eV}\right)^{7 / 2}}{\left(L_{\|} / 1 \mathrm{~m}\right)} \\
\Pi_{T} & \approx 5.810^{-2} \mathrm{Jm}^{-3} \frac{\left(T_{R} / 1 \mathrm{eV}\right)^{3}}{\left(L_{\|} / 1 \mathrm{~m}\right)} \\
\frac{Q_{R}}{c_{z}} & \approx 5.310^{4} \mathrm{Wm}^{-2} \frac{\left(T_{R} / 1 \mathrm{eV}\right)^{4}}{\left(L_{\|} / 1 \mathrm{~m}\right)} \\
n_{T} & \approx 3.610^{-2} 10^{19} \mathrm{~m}^{-3} \frac{\left(T_{R} / 1 \mathrm{eV}\right)^{2}}{\left(L_{\|} / 1 \mathrm{~m}\right)}
\end{aligned}
$$


Finally, using these normalisation rules one can determine the control parameters, $\mathcal{C}_{z}=$ $Q_{R} / Q_{T}, \mathcal{C}_{\pi}=\Pi_{d i v} / \Pi_{T}$ and $Y_{u p}=Q_{u p} / Q_{T}$. with therefore:

$$
\mathcal{C}_{z}=\frac{Q_{R}}{Q_{T}}=58 c_{z}\left(T_{R} / 1 \mathrm{eV}\right)^{1 / 2}
$$

One then finds that increasing $T_{R}$ by a factor 4 , roughly as achieved when switching from carbon $(Z=6)$ to neon $(Z=10)$, would allow one to decrease the impurity concentration by a factor 2 to achieve the same value of the control parameter $\mathcal{C}_{z}$. This drop in impurity concentration is such that the fuel dilution in the core would then be comparable. However, matching an experiment with carbon impurity compared to neon impurity would require increasing the power by a factor 128, eq.( $33 \mathrm{a}$ ) and the plasma pressure by a factor 64 eq. (33b). Conversely, maintaining the injected power and plasma pressure would be equivalent to downshift $Y$ by a factor 128 and the plasma pressure by a factor 64 hence a considerable change in the divertor operation point.

\subsection{Width effect of the radiative cooling rate}

When investigating more realistic radiative cooling rates $\mathcal{L}_{z}$, the main change with respect to the analysis of Section 4 is governed by the width of the radiative function. This effect changes the properties that are governed by the profiles along the direction parallel to the magnetic field: i) the location of the radiative front $z_{R}$, ii) the shape of the radiative work $W$ and its consequence on radiative bifurcations, and iii) the upstream temperature $U_{u p}$ and consequently the upstream density $N_{u p}$ for a given plasma pressure $\mathcal{C}_{\pi}$ or conversely the plasma pressure at given upstream density.

Numerical computation with a realistic shape of a narrow radiative cooling rate provides a first insight into the width effect Fig. 16. The dependence of the radiative layer position on the divertor temperature is found to exhibit the same behaviour as determined in Section 4, Fig. 5. The front width $z_{R}\left(T_{R}+\Delta T_{R}\right)-z_{R}\left(T_{R}-\Delta T_{R}\right)$ is given here by the distance between temperatures that characterise the width of the radiative cooling rate, approximating the peak of the latter by a Gaussian at $T_{R}$ and width $\Delta T_{R}$. One finds that the width of the front does not vary significantly and remains a relatively small fraction of the connection length. Analytical calculations can be performed to investigate this effect when assuming a linear dependence on $X=\left(T / T_{R}\right)^{7 / 2}$ of the work function $w(X)$, eq.(14) Fig. 2, in the transient regime between the saturated work function $w(X)=1$ for $X \leq X_{c}$ and the vanishing value of the work function $W(X)=0$ for $X \geq X_{h}$, hence for $X_{c} \leq X \leq X_{h}$

$$
\begin{aligned}
w(X) & =\frac{X_{h}-X}{\Delta_{X}} \\
\frac{d X}{d z} & =Y_{u p}\left(1-\frac{2 \mathcal{C}_{z} \mathcal{C}_{\pi}}{Y_{u p}^{2}} w(X)\right)^{1 / 2}
\end{aligned}
$$

where $\Delta_{X}=X_{h}-X_{c}$ and $Y_{\text {div }}^{2}=Y_{u p}^{2}-2 \mathcal{C}_{z} \mathcal{C}_{\pi}$. The profile of $X$ is then a quadratic 
function of the normalised curvilinear abscissa $z$.

$$
\begin{aligned}
X & =\frac{1}{2} \frac{Y_{u p}-Y_{d i v}}{z_{h}-z_{c}}\left(z-z_{c}\right)^{2}+Y_{d i v}\left(z-z_{c}\right)+X_{c} \\
Y & =\frac{Y_{u p}-Y_{d i v}}{z_{h}-z_{c}}\left(z-z_{c}\right)+Y_{d i v} \\
\Delta z_{f} & =z_{h}-z_{c}=\frac{2 \Delta_{X}}{Y_{u p}+Y_{d i v}}
\end{aligned}
$$

where $X\left(z_{c}\right)=X_{c}, Y\left(z_{c}\right)=Y_{\text {div }}$ and $X\left(z_{h}\right)=X_{h}, Y\left(z_{h}\right)=Y_{u p}$. Since the decrease of the heat flux through the radiative front must be smaller that the heat flux input $Y_{u p}$, one has $Y_{u p} \leq Y_{u p}+Y_{d i v} \leq 2 Y_{u p}$ so that the width of the radiative front $\Delta z_{f}=z_{h}-z_{c}$ verifies $\Delta_{X} / Y_{u p} \leq \Delta z_{f} \leq 2 \Delta_{X} / Y_{u p}$. In the asymptotic case with vanishing width and peak localised at $X_{R}=X_{c}+\frac{1}{2} \Delta_{X}=X_{h}-\frac{1}{2} \Delta_{X}$, one can compute the distance $\Delta z=z_{h}-z_{c}=\frac{1}{2} \Delta_{X}\left(1 / Y_{u p}+1 / Y_{\text {div }}\right)$ between the points on the temperature profiles at $X_{c}$ and $X_{h}$. One then finds that the front width $\Delta z_{f}$ is smaller than the distance $\Delta z$.

$$
\Delta z_{f}=\frac{2 \Delta_{X}}{Y_{u p}+Y_{d i v}} \leq \frac{\Delta_{X}\left(Y_{u p}+Y_{d i v}\right)}{2 Y_{u p} Y_{d i v}}=\Delta z
$$

this statement being equivalent to $\left(Y_{u p}-Y_{\text {div }}\right)^{2} \geq 0$. Increasing the front width thus also tends to increase the upstream temperature since $X_{u p}=X_{h}+Y_{u p}\left(1-z_{c}-\Delta z_{f}\right) \geq$ $X_{h}+Y_{u p}\left(1-z_{c}-\Delta z\right)$ where $z_{c}=\left(X_{c}-X_{d i v}\right) / Y_{\text {div }}$ does not directly depend on the width of the radiating layer. The effect of a finite front width is thus equivalent to a zero width limit with a slightly increased input power driving an increased midplane temperature, which is favourable by yielding a lower upstream density at prescribed plasma pressure. Unless one addresses really broad cooling rates, such that radiation will escape the divertor volume, one finds that the width effect of the radiative cooling function is not too strong so that the analytical limit at vanishing width still holds.

\subsection{Optimised divertor operation}

The divertor concept must be understood as an interface between the burning plasma located at the centre of the main chamber and plasma facing components with high heat flux extraction capability, the target plates, located in the divertor chamber. In such a framework, line radiation by low Z impurities, intrinsic or seeded impurities, must comply with the role assigned to the divertor. Optimised divertor operation must therefore ensure the synergy between three kinds of constraints.

- Boundary conditions for the core plasma.

- Given output energy flux ( $Q_{u p}$ in the present 1-D model)

- Highest possible boundary plasma pressure (here the upstream value $\Pi$ )

- Controlled upstream density $\left(N_{u p}\right)$ governed by density limit considerations,

- Consequently high upstream plasma temperature $\left(U_{u p}\right)$ to comply high pressure with controlled density.

- Core fuelling in main species and required impurities to balance the particle outflux from the core. 
- Low contamination of the core stemming from wall erosion material like tungsten, seeding impurity for divertor radiation, as well as helium ash recirculation.

- Boundary conditions for the plasma facing components.

- Constrained heat flux to the component $\left(Y_{\text {div }}\right)$ combining steady state operation requirement and smoothed out transients.

- Constrained plasma electron temperature $\left(U_{\text {div }}\right)$ to minimise wall material erosion, by both the main species and impurities in particular seeding impurities.

- Sufficient neutral pressure in the pumping plenum to sustain adequate pumping.

- Stable operation, allowing, as far as possible, a decoupling between plasma conditions at the divertor target plate and upstream conditions.

- Operation without bifurcations or strong variations of the plasma parameters with small changes of the control parameters.

- Appropriate location of the radiating front $\left(z_{R}\right)$

In the model we have singled out four control parameters, $Y_{u p}$ akin to the input power, $\mathcal{C}_{\pi}$ standing for the plasma pressure, $\mathcal{C}_{z}$ the impurity concentration and finally the radiative properties of the species chosen for impurity seeding. One can consider that one has an effective control on each of these parameters but for the plasma pressure, which results from the particle and energy fluxes impinging onto the divertor target plates, see eq.( 10).

$$
\begin{aligned}
Y_{d i v} G_{d i v} & =\frac{1}{2} \mathcal{C}_{\pi}^{2} \\
\frac{Y_{d i v}}{G_{d i v}} & =2 U_{d i v}
\end{aligned}
$$

where $G_{d i v}$ is the normalised particle flux $\Gamma_{d i v}=G_{d i v} N_{T} C_{R}$. The particle flux is the result of complex matter recirculation in the divertor region. It will depend on the neutral pressure in the divertor chamber and can be controlled by feed-back to sustain either a given midplane plasma pressure or a given density. It is to be noted that control of particle recirculation by gas injection and pumping is a non-linear process, with time scales that can be long together with complex transient regimes. Furthermore, the 1-D analysis addressed in this paper does not allow one taking into account profile effects on the target plate. As can readily be seen in eq.(38), the respective location of the peak values of the heat and particle flux is an important issue. Aligning heat and particle flux, hence ensuring their maxima at the same radial location on the target plate appears to be mandatory in terms of divertor performance. Indeed when the maxima of $G_{d i v}$ and $Y_{d i v}$ coincide, one maximises the pressure eq.( $\left.38 \mathrm{a}\right)$ while minimising $U_{\text {div }}$ eq.( $38 \mathrm{~b}$ ) at the location of maximum heat-flux.

In order to analyse divertor operation, we define ratio parameters between characteristic upstream and downstream plasma properties, namely, heat flux, pressure, temperature 
and density in a regime with and without radiation.

$$
\begin{aligned}
N_{u p}=\beta_{N} N_{u p}^{n R} ; \quad \mathcal{C}_{\pi} & =\beta_{\pi} \mathcal{C}_{\pi}^{n R} \\
Y_{u p}=\beta_{Y} Y_{u p}^{n R} ; \quad U_{u p} & =\beta_{U} U_{u p}^{n R} \\
Y_{d i v}=\alpha_{Y} Y_{d i v}^{n R} \quad ; \quad U_{d i v} & =\alpha_{U} U_{d i v}^{n R}
\end{aligned}
$$

where the superscript $n R$ refers to the regime without radiation. Further relations can be introduced for more generality, hence for particle fluxes, ion temperature, or Mach numbers. One could consider total plasma pressure losses along the field line. Within the framework of the present radiative divertor model these parameters are fixed by the assumptions, hence plasma total pressure conservation, vanishing Mach number at the midplane, hence vanishing particle flux, and Mach number \pm 1 at the target plate and finally equal ion and electron temperature. Among the parameters defined in eq.(39), relations exist by definition, hence $\beta_{\pi}=\beta_{N} \beta_{U}$ eq.(26) and similarly for downstream conditions had $\alpha_{N}$ been introduced. The Bohm boundary constraints at Mach \pm 1 introduces another relation: $\alpha_{Y}^{2}=\beta_{\pi}^{2} \alpha_{U}$.

Among the six parameters of eq. (39), we will consider that two, $\beta_{Y}$ and $\alpha_{Y}$, are set by the divertor performance to be achieved, namely the reduction of the heat flux onto the target plate, characterised by $\alpha_{Y}$, with usually prescribed input power, hence $\beta_{Y}=1$. It is also interesting to note that without radiation, we assume no heat flux loss along the field line so that $Y_{u p}^{n R}=Y_{d i v}^{n R}$. Finally, we will consider that the parameter $\beta_{\pi}$ will be constrained by the property of the radiative layer, which we characterise by the input parameter $Y_{u p}^{n R}$ and the position of the radiative layer $z_{R}$, see Section 4.1. Let us first determine $\beta_{U}$ :

$$
\begin{aligned}
U_{u p} & =\left(1+\beta_{Y} Y_{u p}^{n R}\left(1+z_{R}\right)\right)^{2 / 7} \\
U_{u p}^{n R} & =\left(U_{d i v}^{n R^{(7 / 2)}}+Y_{u p}^{n R}\right)^{2 / 7} \\
\beta_{U} & =\left(\frac{1+\beta_{Y} Y_{u p}^{n R}\left(1+z_{R}\right)}{U_{d i v}^{n R^{7 / 2}}+Y_{u p}^{n R}}\right)^{2 / 7}
\end{aligned}
$$

where 1 in eq. (40a) stands for the temperature at the maximum of the radiative cooling rate and $\beta_{Y} Y_{u p}^{n R}$ for the input power with radiation and $0 \leq z_{R} \leq 1$. In eq.( 40b), the unknown $U_{d i v}^{n R}$ is computed given $U_{d i v}^{n R}$ :

$$
U_{d i v}^{n R}=\frac{1}{\alpha_{U}} U_{d i v}=\frac{\beta_{\pi}^{2}}{\alpha_{Y}^{2}}\left(1-\alpha_{Y} Y_{u p}^{n R} z_{R}\right)^{2 / 7}
$$

In a first analysis, see Fig. 17 left hand side, one considers a fixed value of the input power, here $Y_{u p}^{n R} \approx 3.1$ is chosen together with the upstream density with and without radiation, so that $\beta_{N}=1$, and three chosen values of $\alpha_{Y}, \alpha_{Y}=0.1, \alpha_{Y}=0.3$ and $\alpha_{Y}=0.5$, the heat flux ratio into the target plate with and without radiation. For these three values one then plots the pressure ratio $\beta_{\pi}$ versus the location of the radiative front $z_{R}$, Fig. 17, left hand side. One thus finds that impurity seeding at constant upstream density and constant input power governs a decrease of the plasma pressure. The plasma pressure drop is more important when further reducing the target heat flux. It also appears to 
vary rather weakly with the position of the radiative front $z_{R}$. Conversely, one finds that a modest reduction of plasma pressure will tend to yield a large displacement upstream of the radiative front. It is to be noted that requiring a heat flux reduction at the target plate by a factor ten, $\alpha_{Y}=0.1$, in these conditions, will yield a factor two decrease in the plasma pressure, which must impact the fusion performance of the core plasma. The same data can be used to investigate the drop in divertor temperature with impurity seeding, Fig. 17 right hand side, with $Y_{u p}^{n R} \approx 3.1$ and $\beta_{N}=1$. Given $\alpha_{U}=\alpha_{Y}^{2} / \beta_{\pi}^{2}$, one thus finds a large drop of the temperature of the divertor plasma with impurity seeding due to the dependence on $\alpha_{Y}^{2}$. This effect is partly balanced by the drop in pressure that tends to reduce the impurity cooling capability.

This loss of plasma pressure can be compensated by increasing the plasma density, hence increasing $\beta_{N}$ above one, Fig. 17 right hand side, black curves with closed square and circle symbols. In practise this is readily done by injecting a mixture of gaseous impurity and deuterium fuel, a procedure that is used for a better control of the amount impurity introduced in the plasma. Given eq.(38a), an alternative means to compensate the pressure loss is to increase the input power, Fig. 18. Although the observed trend is towards an increase of the plasma pressure, the effect is found to be modest with respect to the target $\beta_{\pi} \approx 1$. Furthermore, operation at higher heat flux but otherwise constant heat flux onto the divertor target plate $\alpha_{Y}=0.1$, then requires a larger impurity injection.

This effect is also noticeable on Fig. 19 right hand side, now considering a fixed location of the radiative front, $z_{R}=0.3$ and varying the input power $Y_{u p}^{n R}$. One then finds that increasing the input power leads to an increase of the plasma pressure, this effect being however less effective than increasing the upstream density $\beta_{N}$, Fig. 19 right hand side. Furthermore, one finds that in all cases with reasonable increase of the input power or of the upstream density, the net effect of impurity seeding is to reduce the plasma pressure, $\beta_{\pi} \leq 1$, hence the fusion performance of the device.

The analysis of the divertor performance that has been presented above has mainly examined the cost of building the radiative layer in terms of plasma pressure. In fact this effect is largely governed by the relation between $\beta_{\pi}, \alpha_{U}$ and $\alpha_{Y}$ since $\beta_{\pi}=\alpha_{Y} / \sqrt{\alpha_{U}}$, see Fig. 19 left hand side. It is then clear that divertor performance characterised by a reduced heat flux to the target plate, and therefore $\alpha_{Y} \rightarrow 0$ will only be consistent with reduced divertor plasma temperature, hence $\alpha_{U} \rightarrow 0$, if the plasma pressure does not decrease, hence for $\alpha_{U} \ll \alpha_{Y}$. However, such a description leaves aside the cost in terms of the amount of injected impurity and in particular the limitation due to fuel dilution in the core plasma. Starting from eq. ( 41) for $U_{d i v}$, one can determine the relation between the reduction of the heat flux to the target plate $\alpha_{Y}$ and the control parameter $\mathcal{C}_{z}$, proportional to the impurity concentration. Given eq.( 20) with $w\left(U_{\text {div }}\right)=1$, one must determine $U_{*}$ for the chosen operation point. Since $U_{*} / U_{\text {div }}=Y_{\text {up }}^{2} / Y_{d i v}^{2}$ and $2 \mathcal{C}_{z}=U_{*} / U_{\text {div }}$, one finds:

$$
\mathcal{C}_{z}=\frac{1}{2}\left(\frac{Y_{u p}^{2}}{Y_{d i v}^{2}}-1\right) U_{d i v}=\frac{1}{2}\left(\frac{\beta_{Y}^{2}}{\alpha_{Y}^{2}}-1\right)\left(1-\alpha_{Y} Y_{u p}^{n R} z_{R}\right)^{2 / 7}
$$

As noticeable when considering the dependence on $\alpha_{Y}$ the required impurity concentration is the balance of two effects: on the one hand, the difference between $Y_{u p}$ and $Y_{d i v}$ that yields the first coefficient and governs an increased impurity concentration to achieve 
the heat flux reduction required by the chosen operation point, on the second hand, the divertor thermal energy $U_{d i v}$, for which the lower $U_{d i v}$, the less impurity concentration is required.

However, it is to be noticed that reducing $U_{\text {div }}$ drives the system towards the point where the radiative regime is lost, $\alpha_{Y} \leq 1 /\left(Y_{u p}^{n R} z_{R}\right)$. Furthermore, one finds that for the standard operation regime $\beta_{Y} \approx 1$ with large radiative losses of heat flux $\alpha_{Y} \ll 1$, the first coefficient becomes very large, leading to large values of the impurity concentration $\mathcal{C}_{z} \gg 1$ except in a narrow region of the operating space with small enough $U_{\text {div }}$. Assuming that reasonable values of $\mathcal{C}_{z}$ are constrained by $\mathcal{C}_{z} \leq 1$ then bounds the value of $\alpha_{Y}$ that can be achieved, typically $\alpha_{Y} \geq 0.5$.

As a consequence, impurity seeding cannot be considered as a stand alone procedure to enter a regime of high divertor performance. First, as just discussed, the drop in heat flux is bounded, second it drives a plasma pressure drop that must be compensated by an enhanced particle recycling flux. The main advantage is to reduce the plasma thermal energy in the divertor. This temperature drop is beneficial to reduce erosion and to enter the detachment regime with appropriate mechanisms to avoid the loss of solution at $\alpha_{Y}^{*}=1 /\left(Y_{u p}^{n R} z_{R}\right)$.

\subsection{Dependence of divertor regimes on connection lengths}

The size effect, eq. (33), is only determined by the dependence on $L_{\|}$of the parameter normalisations. When increased it governs a linear reduction of all these normalisation parameters, and consequently an increase of the effective heat flux and divertor temperature, which is readily translated in terms of a reduction of divertor performance. The dependence on size through the parameter $L_{\|}$is therefore unfavourable for large device operation as well as advanced divertor concepts that aim at increasing the connection length. Furthermore, the dependence on $L_{\|}$is always too small to compensate the very large factors introduced by the change in radiative temperature when changing impurity species. Playing on these two parameters does not provide a flexible way to enlarge the operational space of high performance radiative divertor.

At comparable impurity seeding conditions, same $T_{R}$ and same $\mathcal{C}_{z}$, one finds that in order to operate with the same normalised conditions when increasing $L_{\|}$by a factor 2 would then require a reduction of the input power and plasma pressure by a factor 2 to obtain the appropriate matching. Alternatively, one can proceed as in Section 5.3 but comparing cases with two different values of the parameter $L_{\|}$with a list of coefficients that take into account the ratio of the control parameter values for these two cases as done for the radiating and non-radiating case, eq.( 39$)$.

$$
\begin{aligned}
N_{u p}=\beta_{N} N_{u p}^{r e f} ; \quad \mathcal{C}_{\pi} & =\beta_{\pi} \mathcal{C}_{\pi}^{r e f} \\
Y_{u p} & =\beta_{Y} Y_{u p}^{r e f} ; \quad U_{u p}=\beta_{U} U_{u p}^{r e f} \\
Y_{d i v} & =\alpha_{Y} Y_{\text {div }}^{r e f} ; \quad U_{d i v}=\alpha_{U} U_{d i v}^{r e f}
\end{aligned}
$$

where the superscript ref now refers to the reference case with connection length $L_{r e f}$ that is compared to another case with different connection length, corresponding to the values with no superscript. Some relations between the coefficients are imposed by the definitions of the chosen control parameters, hence $\beta_{\pi}=\beta_{N} \beta_{U}$ (similarly, one would have $\alpha_{\pi}=\alpha_{N} \alpha_{U}$ ), or by the Bohm boundary conditions, assuming a Mach one condition, $\alpha_{U}=\alpha_{Y}^{2} / \alpha_{\pi}^{2}$. Finally other relations are governed by the chosen physics, hence $\alpha_{\pi}=\beta_{\pi}$ 
for a case without parallel pressure loss. Given the change in geometry, one must also allow for a change in the location of the radiative front, hence:

$$
z_{R}=\alpha_{z} z_{R}^{r e f}
$$

One therefore obtains a new relation for the divertor thermal energy:

$$
\begin{aligned}
U_{d i v}^{r e f} & =\left(1-Y_{d i v}^{r e f} z_{R}^{r e f}\right)^{2 / 7}=\left(1-\alpha_{Y}^{r e f} Y_{u p}^{r e f} z_{R}^{r e f}\right)^{2 / 7} \\
U_{d i v} & =\left(1-\beta_{Y} \alpha_{z} \alpha_{Y}^{r e f} Y_{u p}^{r e f} z_{R}^{r e f}\right)^{2 / 7} \\
\alpha_{U} & =\left(\frac{1-\beta_{Y} \alpha_{z} \alpha_{Y}^{r e f} Y_{u p}^{r e f} z_{R}^{r e f}}{1-\alpha_{Y}^{r e f} Y_{u p}^{r e f} z_{R}^{r e f}}\right)^{2 / 7}
\end{aligned}
$$

where $\alpha_{Y}^{r e f}$ is the performance index of the reference scenario in terms of heat flux reduction at the target plate with respect to a case without radiation, $\alpha_{Y}^{r e f}=Y_{d i v}^{r e f} / Y_{u p}^{r e f}$. In a matching set of experiments comparing solutions with different connection lengths, at comparable core confinement, one will tend to keep the injected power constant as well as the core density, insofar that one can change the connection length without changing the magnetic geometry of the core plasma.

Given the change in normalisation of the heat flux, one then finds that the parameter $\beta_{Y}$ accounts for the change in connection length $\beta_{Y}=L_{\|} / L_{\text {ref }}$ at constant input energy flux ( $L_{r e f}$ is the reference connection length). With respect to the reference case, one thus finds that increasing $\beta_{Y} \alpha_{z}$ drives the divertor temperature $U_{\text {div }}$, eq. (45b), closer to the limit. Indeed, one now requires $\alpha_{Y}^{r e f} Y_{u p}^{r e f} z_{R}^{r e f} \leq 1 /\left(\beta_{Y} \alpha_{z}\right)$, compared to the reference case $\alpha_{Y}^{r e f} Y_{u p}^{r e f} z_{R}^{r e f} \leq 1$. Transport effects with an enhanced connection length thus tend to yield $\alpha_{U} \leq 1$, Fig. 21. In fact one recovers the expected result, namely that iso-values of $\alpha_{U}$ are determined by $\left(z_{R} / z_{r e f}\right)\left(L_{\|} / L_{r e f}\right)=c s t$. Reducing this product governs an increase of $\alpha_{U}$. Thus depending on the change of geometry, one can find a localisation of the radiation front such that the temperature at the divertor decreases, which is a favourable trend.

For the upstream temperature one finds:

$$
\begin{aligned}
U_{u p}^{r e f} & =\left(1+Y_{u p}^{r e f}\left(1-z_{R}^{r e f}\right)\right)^{2 / 7} \\
U_{u p} & =\left(1+\beta_{Y} Y_{u p}^{r e f}\left(1-\alpha_{z} z_{R}^{r e f}\right)\right)^{2 / 7} \\
\beta_{U} & =\left(\frac{1+\beta_{Y} Y_{u p}^{r e f}\left(1-\alpha_{z} z_{R}^{r e f}\right)}{1+Y_{u p}^{r e f}\left(1-z_{R}^{r e f}\right)}\right)^{2 / 7}
\end{aligned}
$$

and a trend towards $\beta_{U} \geq 1$ for $\beta_{Y} \geq 1$ is also governed by transport properties along the long field line. Hence the effect of increasing $\beta_{Y}=L_{\|} / L_{\text {ref }}$ drives an increase of $\beta_{U}$, and, increasing $z_{R} / z_{\text {ref }}$ governs a similar trend but with a less marked effect.

Given $\beta_{U}$ and $\beta_{N}$, one then readily determines $\beta_{\pi}$ and consequently $\alpha_{Y}$, Fig. 21. Since $\alpha_{Y}$ includes the effect of the change in connection length in its normalisation, we thus consider $\alpha_{Y} / \beta_{Y}$ to estimate the actual impact of changing the connection length in terms 
of heat flux deposition onto the target plate. The region without solution, where $\alpha_{Y} / \beta_{Y}$ is set at zero on Fig. 21, is imposed by the region with no solution for $\alpha_{U}$ in eq.( $\left.45 \mathrm{c}\right)$. One finds that iso-contours exhibit a maximum that shifts towards the small values of $\beta_{Y}$ for decreasing values of $\alpha_{Y} / \beta_{Y}$. The main trend is that increasing $L_{\|}$tends to govern an increase in the heat flux deposition except in a narrow region close to the limit $U_{d i v} \rightarrow 0$.

The normalisation of the impurity concentration eq.( 34) does not exhibit an explicit dependence on the connection length. However, when matching the reference case to the operation point with different connection lengths, one is led to modify various control parameter so that a change in impurity concentration $\mathcal{C}_{z}$ is required for consistency. Considering eq.( 42), one then obtains:

$$
\mathcal{C}_{z}=\alpha_{U}\left(\frac{\left(\beta_{Y}^{2} / \alpha_{Y}^{2}\right)-\alpha_{Y}^{r e f^{2}}}{1-\alpha_{Y}^{r e f^{2}}}\right) \mathcal{C}_{z}^{r e f}
$$

In a matching experiment, lower impurity concentration is then required as one increases the connection length $L_{\|}$. This is possible with impurity seeding, and unlikely achieved with intrinsic impurities. If the reference case is without impurity seeding, one thus finds that a matching experiment with a large change in $L_{\|}$under comparable conditions is not possible. Conversely, when reducing the connection length, matching experiments then require enhanced impurity concentration. The change in divertor operating point will therefore impact other aspects of plasma-wall interaction. One also finds that when $L_{\|}$is increased the operating window closes, one boundary being governed by the limit $U_{\text {div }} \rightarrow 0$, the other by the line of zeros impurity concentration, Fig. 22 left hand side. This narrowing aspect of the operation window also drives the operating point towards a modification of the radiating front location, with $\alpha_{z} \approx 0.25$ asymptotically which yields $z_{R}=\alpha_{z} z_{\text {ref }} \approx 7.510^{-2}$. Consequently, the change in geometry leading to the increase in connection length does not appear to readily govern an increase of the divertor volume with cold plasma. The overall effect thus appears to be rather modest in contrast to the large change in plasma geometry, and consequently in device geometry, required to change the connection length.

As a final illustration of the effect of changing the connection length in the present radiative divertor model we consider matching experiments at radiation front location $z_{R}$ fixed with $\alpha_{z}=0.5$ and consequently $z_{R}=0.15$ given the chosen value of $z_{\text {ref }}=0.3$, Fig. 22 right hand side. One finds that matching parameters are kept roughly constant, in particular the plasma pressure (blue curve, open circles, right hand side scale) which plays an important role in the radiative losses as well as regarding the boundary conditions. The divertor heat flux exhibits the largest increase with connection length although the trend appears to reverse in the vicinity of the limit $U_{d i v} \rightarrow 0$, which bounds $L_{\|}$given the chosen value of $z_{R}$.

\section{Discussion and conclusion}

Several remarkable properties of fusion plasmas confined by strong magnetic fields appear to be governed by self-organisation. As an example of such aspects, the effect of line radiation governed by light impurity seeding on the issue of heat exhaust by the divertor 
are presented in this paper. This example is also well suited to show how technical constraints require novel physics to control the operation point at highest fusion performance. Indeed, the operation constraints that result from heating matter in the plasma state at temperatures higher than that experienced in the sun, albeit at lower densities, call for numerous trade-off between technology and physics.

To address this difficult issue, we have chosen a simplified model that allows one to solve the problem analytically. Despite the numerous issues raised by the various simplifications, the model also allows one to carry out a complete analysis that can be used as a guide line for more advanced modelling, requiring numerical simulations. The input is two fold: the working assumptions are clearly identified and can therefore be tested, the complex self-organised properties of the non-linear physics at hand, such as bifurcations and operation in a large space of control parameters, provides a way to analyse and understand the results of these more complete investigations.

The model at hand is a 1-D model in the direction parallel to the magnetic field, and addresses heat transport of a single species plasma. The latter can be viewed either as a one fluid model of plasma, hence assuming strong equipartition between ion and electron temperatures with heat diffusion dominated by electron transport, the chosen assumption of the present paper, or only electrons in the opposite limit of vanishing equipartition between ion and electron temperatures. The latter model in the cold ion limit would change some numerical parameters in the model but yield qualitatively the same results.

As in many analytical approximations addressing heat transport and temperature profiles, one bypasses the issue of particle transport by considering the mechanical constraint on the plasma, in particular the pressure balance along the field lines, and by assuming that the particle flux build-up occurs in a narrow region located in the very vicinity of the target plates. The relation with the plasma density is only introduced when considering various control strategies, usually fixed midplane properties, either plasma pressure or density. This assumption is a strong limitation of the model together with neglecting cross-field transport that can be strongly modified in the divertor volume and consequently play a more important role that would stem from standard considerations on the SOL width (the smaller the SOL heat channel the more transport is dominated by parallel transport).

Combined with the Bohm conditions, the temperature and heat flux profiles then appears to result from a Hamiltonian system such that temperature and heat flux are conjugate variables, the curvilinear abscissa being the time variable. The Hamiltonian is then the sum of a kinetic energy term, the momentum being the heat flux and a radiative work function depending on temperature akin to a the location in space. The radiative work or radiative potential energy is the integral of the radiative cooling rate. It is thus found to depend on the chosen impurity but many details of the cooling rate function are smoothed out by the integration. For a narrow radiative cooling function, the radiative work takes the form of a step function. Several key properties are then readily determined, such as the possibility of thermal bifurcation with multiple solutions or critical values of the control parameters for regions without solutions. Although not addressed in this paper, the Hamiltonian formalism should prove a useful tool in analysing the stability of the various trajectories in the case of multiple solutions.

In a generic way one finds that three regimes can be identified: the hot divertor regime, the radiative regime, and when radiation extends out of the divertor volume into the whole SOL, the cold SOL regime. While the former transition is characterised by a bifurcation, 
the latter change in regime is a gradual modification of properties from a situation that is considered as optimum for divertor performance to a situation where radiation expands towards the core plasma, which is considered as deleterious. The bifurcation aspect from the hot divertor to the radiative divertor regime, exhibits interesting properties when considering the $\mathrm{H}$-mode bifurcation. Although it does not exhibit any feature related to confinement improvement that is dear to the $\mathrm{H}$-mode scenario, it can be regarded as a trigger of the transition, favouring conditions that drive a change in turbulent cross-field transport. The latter point remains to be investigated. However, it is clear that many properties associated to the H-mode transition, in particular the role of impurities and correlation with the divertor state, would then be more easily integrated in our understanding. The H-mode transition or at least some of the $\mathrm{H}$-mode transitions would then result from a dual bifurcation, a first bifurcation into the hot divertor regime with a marked change in upstream plasma collisionality, and induced by this change, a bifurcation in turbulent transport properties.

With respect to earlier papers on the radiative divertor, the present analytical work extends the results in two directions, first the location of the radiative front is introduced since the analytical work is complete and the profiles can be determined, second one can investigate the role of the upstream temperature and therefore introduce the cold SOL regime. Both aspects are shown to reduce the operating window and increase the constraints on radiative divertor operation. An illustration of these features is obtained in the modified density regimes. Depending on the peak temperature of the impurity cooling rate, one finds that radiation either occurs in the linear (or sheath limited regime), terminating this regime at lower density, or in the high recycling regime where it favours a rapid drop of the divertor temperature but tends to govern a loss of the solution at large enough heat flux in the system. Analysing the benefit of radiation then crucially depends on the physics that take place when the solution is lost. The alternative could be either a transition of the form of a radiative density limit triggered by divertor conditions or a transition to plasma detachment and the continuation of divertor operation with completely modified particle recirculation. The investigation of plasma detachment thus appears as crucial in determining the benefit of impurity seeding and radiative divertor operation. This analysis is also most important to determine if the two terms of the alternative are effectively independent, or two successive steps of a single transient leading to plasma termination by a radiative collapse.

In the last Section of the paper, we address the important issue of divertor control. A key result is that radiative divertor operation tends to govern a decrease of plasma pressure. The key formula underlying this result stems from the invariant, namely the Hamiltonian of the system that can be recast as $U_{*}=U_{\text {div }} \Pi_{\text {div }}^{2} / \Pi_{u p}^{2}+2 \mathcal{C}_{z} \Pi_{R}^{2} / \Pi_{u p}^{2}$, see eq.( 15c), eq.( 16) and eq.( 22b). However, with respect to these expressions we have not simplified the system assuming a constant pressure along the field line. The first term is the divertor temperature that would be achieved without line radiation and without momentum loss along the field line, hence related to the upstream heat flux by a Bohm conditions $Y_{u p}^{2}=\Pi_{u p}^{2} U_{*}$. The second term is the divertor temperature related to the divertor heat flux according to $Y_{d i v}^{2}=\Pi_{d i v}^{2} U_{d i v}$ derived from Bohm boundary conditions. The last term stands for the radiative loss proportional to the impurity concentration parameter $\mathcal{C}_{z}$ and to the pressure at the radiating front $\Pi_{R}$ squared. Maximising the 
radiative losses is then achieved with the pressure balance between the radiative front and the midplane $\Pi_{R}=\Pi_{u p}$. The condensation mechanism, namely the enhanced density of the species generating the radiative losses, and therefore the radiative losses, as the temperature is decreases, is then maximised. Conversely, one finds that divertor operation is significantly eased with $\Pi_{d i v} \leq \Pi_{u p}$ hence with pressure imbalance between the radiative front and the divertor target plate. The benefit is then two-fold: on the one hand the heat flux to the target plate will result from both a decrease of the plasma pressure and of the plasma temperature, and on the other hand, maximising the radiative efficiency does not impose a working point at the limit $U_{d i v} \rightarrow 0$. Similarly, when operating with stiff conditions governed by pressure balance, the operating window is in fact opened by allowing a pressure drop when comparing non-radiating and radiative operation. This property is found to be governed by the alignment of the heat flux and particle flux that maximises the plasma pressure. In order to sustain a constant pressure, when the divertor heat flux is decreased due to radiation, one must increase the particle flux to the divertor target plate. The latter mechanism then drives the plasma temperature closer to the radiative collapse limit $U_{d i v} \rightarrow 0$. For these reasons, one finds that the radiative divertor, hence divertor operation with seeded impurities, must be considered as a way to enter the detached regime. Indeed, radiation alone has limited impact on the divertor heat flux and a more important effect in reducing the divertor temperature, and consequently reduced divertor performance due to lower plasma pressure or operation close to the radiative collapse. Detachment, the occurrence of a pressure drop between the radiative front and the divertor target plate, then appears as a key mechanism to reduce the heat flux onto the target plate. Provided, such a regime is sufficiently stable, one can then consider that the radiative divertor is appropriate to facilitate the transition from the high recycling radiative divertor to the detached divertor. The complexity introduced by radiative divertor operation is therefore found to be of interest only as a means to reach another self-organised state of the divertor plasma.

\section{Acknowledgements}

This work has been carried out thanks to the support of the A*MIDEX project (ANR-11IDEX-0001-02) funded by the "Investissements d'Avenir" French Government program, managed by the French National Research Agency (ANR).

\section{References}

[1] C. Breton, C. De Michelis, and M. Mattioli. Radiation losses from oxygen and iron impurities in a high-temperature plasma. Nuclear Fusion, 16(6):891, 1976.

[2] H. Capes, Ph. Ghendrih, and A. Samain. Radiative instability in a diverted plasma. Physics of Fluids B, 4(5):1287-1293, 1992.

[3] P. G. Carolan and V. A. Piotrowicz. The behaviour of impurities out of coronal equilibrium. PP, 25(10):1065, 1983.

[4] Ph Ghendrih, K Bodi, H Bufferand, G Chiavassa, G Ciraolo, N Fedorczak, L Isoardi, A Paredes, Y Sarazin, E Serre, F Schwander, and P Tamain. Transition to supersonic flows in the edge plasma. Plasma Physics and Controlled Fusion, 53(5):054019, 2011. 
[5] Ph. Ghendrih, A. Grosman, J. Gunn, F. Laugier, B. Meslin, C. Grisolia, R. Guirlet, P. Monier-Garbet, and T. Loarer. Comparison of ergodic and axisymmetric divertors. Journal of Nuclear Materials, 266-269:189 - 196, 1999.

[6] M. Greenwald, J.L. Terry, S.M. Wolfe, S. Ejima, M.G. Bell, S.M. Kaye, and G.H. Neilson. A new look at density limits in tokamaks. Nuclear Fusion, 28(12):2199, 1988.

[7] A. Loarte, B. Lipschultz, A.S. Kukushkin, G.F. Matthews, P.C. Stangeby, N. Asakura, G.F. Counsell, G. Federici, A. Kallenbach, K. Krieger, A. Mahdavi, V. Philipps, D. Reiter, J. Roth, J. Strachan, D. Whyte, R. Doerner, T. Eich, W. Fundamenski, A. Herrmann, M. Fenstermacher, P. Ghendrih, M. Groth, A. Kirschner, S. Konoshima, B. LaBombard, P. Lang, A.W. Leonard, P. Monier-Garbet, R. Neu, H. Pacher, B. Pegourie, R.A. Pitts, S. Takamura, J. Terry, E. Tsitrone, the ITPA Scrape-off Layer, and Divertor Physics Topical Group. Chapter 4: Power and particle control. Nuclear Fusion, 47(6):S203, 2007.

[8] G F Matthews, M Beurskens, S Brezinsek, M Groth, E Joffrin, A Loving, M Kear, M-L Mayoral, R Neu, P Prior, V Riccardo, F Rimini, M Rubel, G Sips, E Villedieu, $\mathrm{P}$ de Vries, M L Watkins, and EFDA-JET contributors. Jet iter-like wall-overview and experimental programme. Physica Scripta, 2011(T145):014001, 2011.

[9] M. Shimada, D.J. Campbell, V. Mukhovatov, M. Fujiwara, N. Kirneva, K. Lackner, M. Nagami, V.D. Pustovitov, N. Uckan, J. Wesley, N. Asakura, A.E. Costley, A.J.H. Donné, E.J. Doyle, A. Fasoli, C. Gormezano, Y. Gribov, O. Gruber, T.C. Hender, W. Houlberg, S. Ide, Y. Kamada, A. Leonard, B. Lipschultz, A. Loarte, K. Miyamoto, V. Mukhovatov, T.H. Osborne, A. Polevoi, and A.C.C. Sips. Chapter 1: Overview and summary. Nuclear Fusion, 47(6):S1, 2007.

[10] P. C. Stangeby. The plasma boundary of magnetic fusion devices. Plasma physics series. Taylor \& Francis, New York, 2000.

[11] F Wagner. A quarter-century of h-mode studies. Plasma Physics and Controlled Fusion, 49(12B):B1, 2007. 

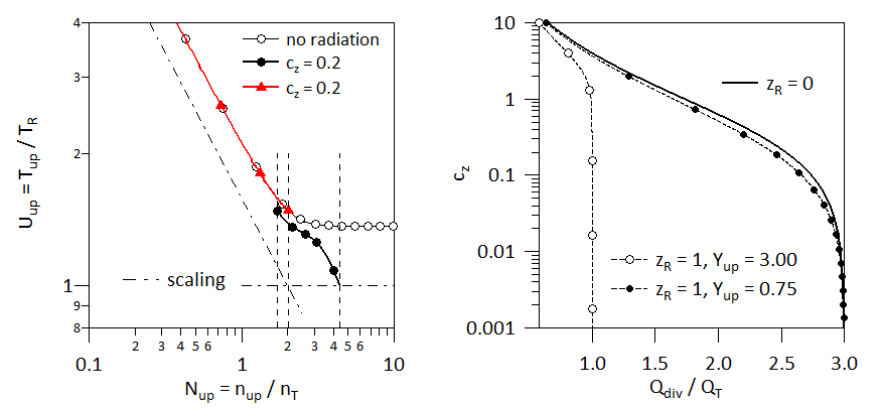

Figure 13: Left hand side upstream temperature versus upstream density at fixed input energy flux $Y_{u p}=3.00$, without radiation open circles, with radiation closed symbols. Right hand side, location of the radiative front $z_{R}$ in the parameter space $\left(Y_{\text {div }}=Q_{\text {div }} / Q_{T}\right.$, $\mathcal{C}_{z}$ ) for two values of the input energy flux $Y_{u p}=0.75$ and $Y_{u p}=3.00$ : plain line without symbols, $z_{R}=0$, closed circles $Y_{u p}=0.75$, open circles $Y_{u p}=3.00$.
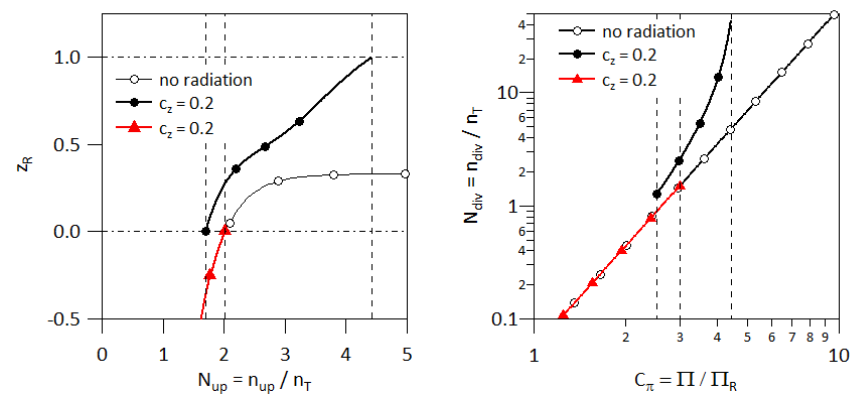

Figure 14: Divertor regimes at fixed input energy flux $Y_{u p}=3.00$, left hand side location of the radiative front $z_{R}$ versus upstream density, right hand side divertor density versus plasma total pressure, without radiation open circles, with radiation closed symbols.
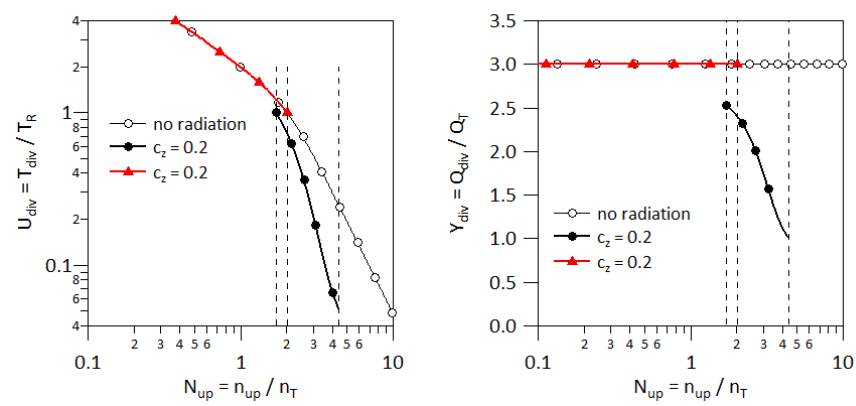

Figure 15: Divertor density regimes at fixed input energy flux $Y_{u p}=3.00$, divertor temperature, left hand side, heat flux to the divertor, right hand side versus upstream density, without radiation open circles, with radiation closed symbols. 


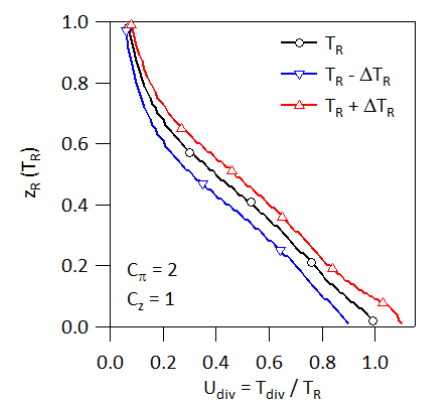

Figure 16: Radiative front location $z_{R}$ versus the divertor temperature $U_{\text {div }}$ in the case of a narrow radiative cooling rate peaked at $T=T_{R}$ and finite width from $T_{R}-\Delta T_{R}$ to $T_{R}+\Delta T_{R}$ at given control parameters, $\mathcal{C}_{\pi}=2$ for the plasma pressure and $\mathcal{C}_{z}=1$ for the impurity concentration.
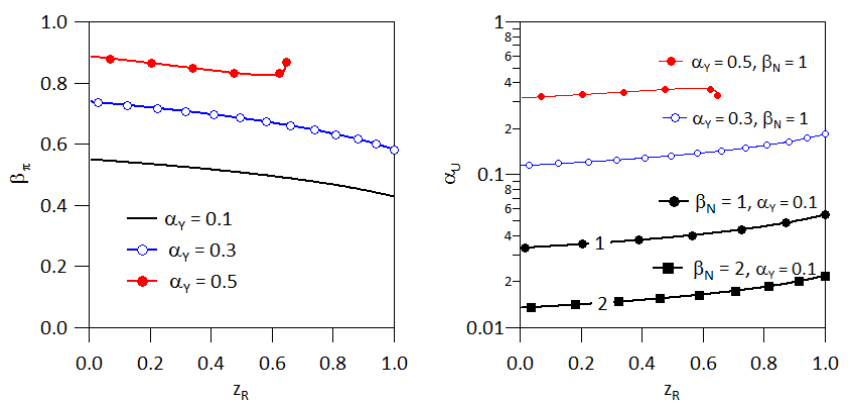

Figure 17: Left hand side figure : divertor operation control at given input energy flux, plasma pressure variation versus the location of the radiative front for three ratios of the divertor heat flux with respect to the value achieved without radiation, $\alpha_{Y}=0.1$, $\alpha_{Y}=0.3$ and $\alpha_{Y}=0.5$. Right hand side figure: plasma temperature variation at the divertor versus the location of the radiative front for the same three ratios of the divertor heat flux with respect to the value achieved without radiation, and two values of the upstream density, $\beta_{N}=1$, and $\beta_{N}=2$.

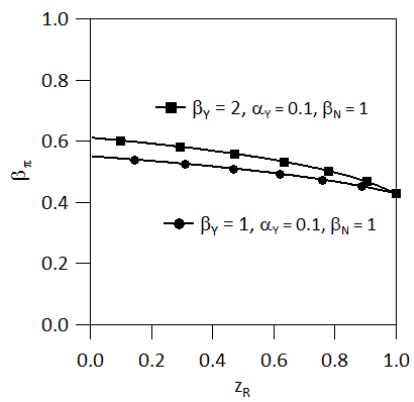

Figure 18: Pressure recovery parameter $\beta_{\pi}$ at fixed upstream density $\beta_{N}=1$ and divertor heat flux $\alpha_{Y}=0.1$ versus the radiative front location $z_{R}$ for two values of the upstream energy flux injection: $\beta_{Y}=1$ closed circles and $\beta_{Y}=2$, closed squares. 

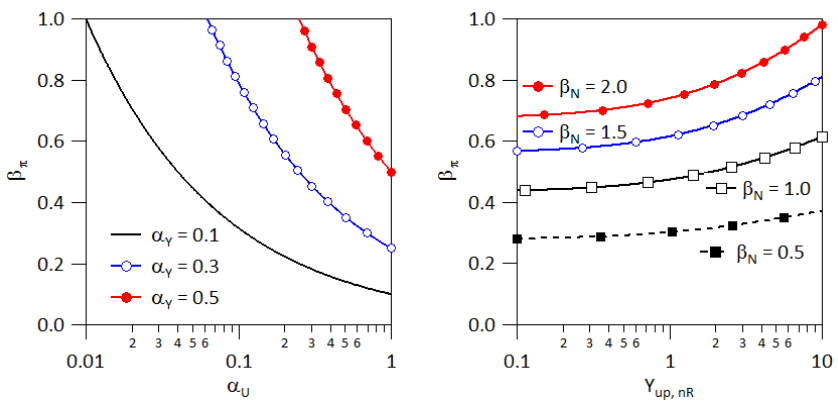

Figure 19: Left hand side: pressure variation parameter $\beta_{\pi}=\alpha_{Y} / \sqrt{\alpha_{U}}$ at fixed upstream density $\beta_{N}=1$ and divertor heat flux $\alpha_{Y}=0.1$ versus the divertor temperature parameter $\alpha_{U}$ for tree values of the divertor heat flux parameter: $\alpha_{Y}=0.1$ plain line, $\alpha_{Y}=0.3$ plain line open circles and $\alpha_{Y}=0.5$, plain line closed circles. Right hand side, pressure recovery parameter $\beta_{\pi}$ versus the energy influx $Y_{u p, n R}$ at given location of the radiative front $z_{R}=0.3$ and different enhancement values of the upstream density: $\beta_{N}=0.5$ closed square markers, $\beta_{N}=1.0$ open squares, $\beta_{N}=1.5$ open circles and $\beta_{N}=1.5$ closed circles.
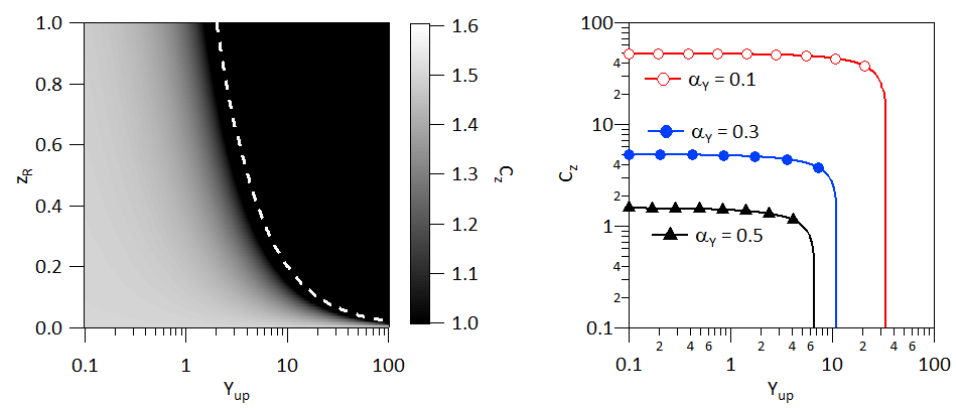

Figure 20: Divertor density regimes at fixed input energy flux $Y_{u p}=3.00$, divertor temperature, left hand side, heat flux to the divertor, right hand side versus upstream density, without radiation open circles, with radiation closed symbols. 

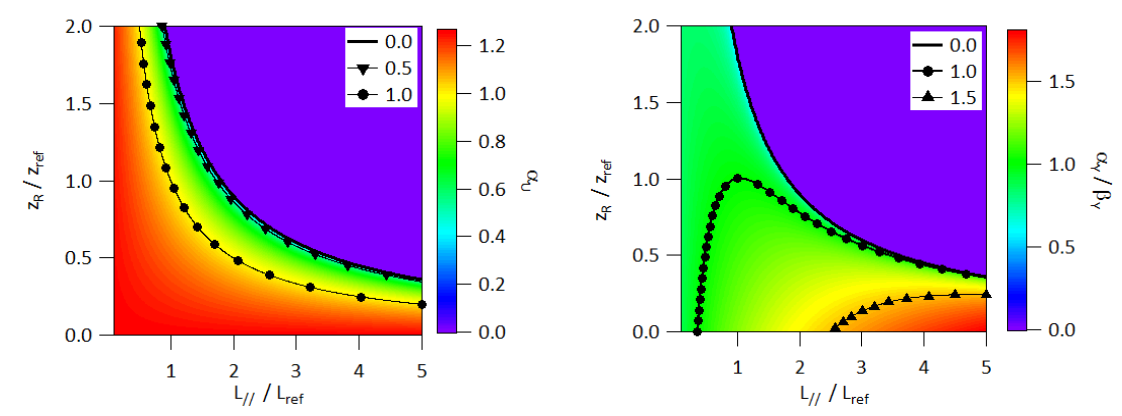

Figure 21: Left hand side, variation parameter of the divertor temperature, $\alpha_{U}$, in the plane $\alpha_{z}=z_{R} / z_{R}^{r e f}, \beta_{Y}=L_{\|} / L_{r e f}$. The plain line $\alpha_{U}=0$ determines the region with solutions, the region with $\alpha_{U}$ ranging from 0 to 1 , plain line closed circles and yellow contour, being those of improved divertor performance. Note the small operation window between the contour for $\alpha_{U}=0.3$, plain line closed triangles, and $\alpha_{U}=0$. Right hand side, variation of the parameter $\alpha_{Y} / \beta_{Y}$, hence the ratio of divertor to upstream heat flux, in the same parameter space $\alpha_{z}=z_{R} / z_{R}^{r e f}$ versus $\beta_{Y}=L_{\|} / L_{r e f}$.
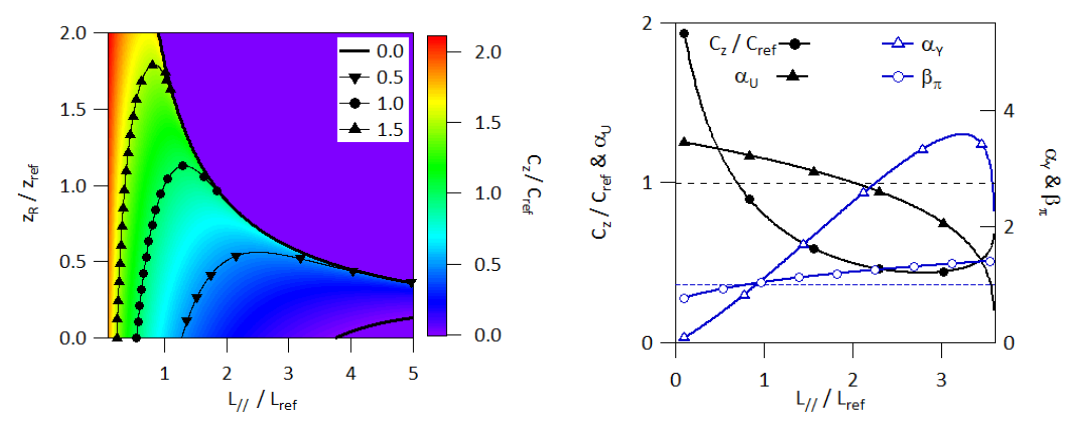

Figure 22: Left hand side: Change in impurity concentration $\mathcal{C}_{z} / \mathcal{C}_{\text {ref }}$ in the parameter space $\alpha_{z}=z_{R} / z_{R}^{r e f}, \beta_{Y}=L_{\|} / L_{r e f}$, four contour lines are highlighted, the boundary line $\mathcal{C}_{z} / \mathcal{C}_{\text {ref }}=0$, plain line, $\mathcal{C}_{z} / \mathcal{C}_{\text {ref }}=0.5$ closed downward triangles, $\mathcal{C}_{z} / \mathcal{C}_{\text {ref }}=1$ closed circles, $\mathcal{C}_{z} / \mathcal{C}_{\text {ref }}=1.5$ closed upward triangle. Right hand side: $\mathcal{C}_{z} / \mathcal{C}_{\text {ref }}$, closed circles, and $\alpha_{u}$, closed upward triangle, left hand side scale and $\alpha_{Y}$, open upward triangles, and $\beta_{\pi}$ open circles, right hand side scale. 